The Astronomical Journal, 118:2014-2037, 1999 November

\title{
A CATALOG OF NEARBY POOR CLUSTERS OF GALAXIES
}

\author{
RICHARD A. WhITE \\ NASA Goddard Space Flight Center, Code 631, Greenbelt, MD 20771 \\ MARK BLITON ${ }^{1}$ \\ Department of Astronomy, New Mexico State University, P.O. Box 30001, Department 4500, Las Cruces, NM 88003 \\ SUKETU P. BHAVSAR \\ Department of Physics and Astronomy, University of Kentucky, Lexington, KY 40506 \\ PATRICIA BORNMANN \\ NOAA Space Environment Center, 325 Broadway, Boulder, CO 80303 \\ JACK O. BURNS ${ }^{2}$ \\ Department of Physics and Astronomy, University of Missouri, Columbia, MO 65211 \\ Michael J. LeDLOW \\ Department of Physics and Astronomy, University of New Mexico, 800 Yale Boulevard NE, Albuquerque, NM 87131 \\ AND \\ CHRISTEN LOKEN \\ Department of Physics and Astronomy, University of Missouri, Columbia, MO 65211 \\ Received 1999 June 22; accepted 1999 July 20
}

\begin{abstract}
A catalog of 732 optically selected, nearby poor clusters of galaxies covering the entire sky north of $-3^{\circ}$ declination is presented. The poor clusters, called WBL clusters, were identified as concentrations of three or more galaxies with photographic magnitudes brighter than 15.7 , possessing a galaxy surface overdensity of $10^{4 / 3}$. These criteria are consistent with those used in the identification of the original Yerkes poor clusters, and this new catalog substantially increases the sample size of such objects. These poor clusters cover the entire range of galaxy associations up to and including Abell clusters, systematically including poor and rich galaxy systems spanning over 3 orders of magnitude in the cluster mass function. As a result, this new catalog contains a greater diversity of richness and structures than other group catalogs, such as the Hickson and Yerkes catalogs. The information on individual galaxies includes redshifts and cross-references to other galaxy catalogs. The entries for the clusters include redshift (where available) and cross-references to other group and cluster catalogs.
\end{abstract}

Key words: catalogs - galaxies: clusters: general

\section{INTRODUCTION}

Traditionally, the study of galaxy associations has focused on rich clusters of galaxies such as the Abell clusters (Abell 1958; Abell, Corwin, \& Olowin 1989). However, these clusters represent the extreme in galaxy associations and, although massive, are relatively rare. Conversely, poor clusters are less massive but more numerous and constitute a significant fraction of the mass of the universe. As the building blocks of clusters and superclusters, poorer galaxy associations must be studied in order to understand the formation and evolution of large-scale structure in the universe. Poor clusters are fundamental entities, but until recently, they have not received as much attention as they merit. This stems in part from a paucity of catalogs that reach, in a consistent way, out to Abell cluster distances.

There are several poor-cluster catalogs in the literature that have yielded important scientific results. The CfA group catalog (Geller \& Huchra 1983), based on both redshift and spatial information (thereby removing the possibility of projection effects). showed groups to be important in tracing large-scale structure. The Hickson compact

\footnotetext{
${ }^{1}$ Current address: Department of Physics and Astronomy, University of Missouri, Columbia, MO 65211.

${ }^{2}$ Office of Research, University of Missouri, Columbia, MO 65211.
}

group catalog (HCG; Hickson 1982), with its high galaxy density criterion, has proved an important resource for studying interacting galaxies. The AWM (Albert, White, \& Morgan 1977), MKW (Morgan, Kayser, \& White 1975), and WP (White 1978) clusters, also known as Yerkes clusters, specifically targeted poor clusters containing $\mathrm{cD}$ galaxies. These clusters have been studied extensively in the optical, radio, and X-ray regions of the spectrum. They possess optical structures that are a continuation of the Abell clusters to lower richness levels (Bahcall 1980), and their X-ray and radio properties show surprising similarities to the Abell clusters as well (e.g., X-ray cooling flows and tailed radio sources; Doe et al. 1995; Burns et al. 1987).

The original identifications of the Yerkes clusters were made by eye in a laborious procedure of scanning the POSS glass plates in conjunction with the Zwicky catalog (CGCG; Zwicky et al. 1961-1968). The new study presented in this paper was undertaken to create a much larger sample of clusters electronically (with galaxy overdensities similar to the Yerkes clusters), utilizing an algorithm that reproduced the human-eye search.

The terms "poor galaxy cluster" and "galaxy group" have not been consistently defined or applied in the literature. Since this catalog includes galaxy associations of all richness levels (see, e.g., Bhavsar 1981), we use the term "poor cluster" to describe these entries. Imposing an arbitrary change in nomenclature, calling rich associations clus- 
ters and poor associations groups, would obscure the continuous spectrum of properties possessed by these objects.

\section{TECHNIQUES}

The creation of the catalog employed the Turner \& Gott (1976, hereafter TG) algorithm, which is capable of mimicking, in a very mathematical, reproducible, and consistent way, the work of the human eye in picking out density enhancements from the POSS. We chose to apply the TG algorithm to the CGCG catalog, since this procedure accomplished our goal of identifying the majority of the Yerkes clusters. The CGCG is a compilation of galaxies and clusters, covering the sky north of $-3^{\circ}$ declination. Zwicky identified galaxies to a limiting photographic magnitude $m_{\mathrm{pg}}=15.7$ on the POSS photographic plates. Zwicky clusters were identified as galaxy associations containing at least 50 galaxies in the magnitude range $m_{\max }$ to $m_{\max }+3$, where $m_{\max }$ is the magnitude of the brightest cluster member. To create this poor-cluster catalog, we used CGCG galaxies as faint as $m_{\mathrm{pg}}=15.7$, which is $0.7 \mathrm{mag}$ deeper than previous poor-cluster searches (e.g., Bhavsar 1980).

The TG algorithm begins with an assigned target factor for galaxy surface density enhancement $\left(\sigma_{g}\right)$. Around each galaxy in the catalog, it determines the smallest circular aperture on the sky, centered on the galaxy, that yields the desired $\sigma_{g}$. The number of additional galaxies, if any, within each aperture is also noted, which we define to be the number of "nearest neighbors." All overlapping apertures are then merged together into a cluster. Galaxies that have no nearest neighbors and are not included within the apertures of other galaxies are considered isolated. The process is then repeated, at a larger value of $\sigma_{g}$, excluding all isolated galaxies. This results in a hierarchy of clusters that shows a given cluster's fragmentation or continued integrity with increasing $\sigma_{g}$. A more detailed discussion of the algorithm and its operation may be found in earlier papers (TG; Bhavsar \& Piggott 1983; Bhavsar 1980).

Levels of $\sigma_{g}$ were chosen to be multiplicative factors above the average surface density of CGCG galaxies determined in the region of the Galactic caps $\left(|b|>40^{\circ}, \delta>0^{\circ}\right.$; 6866 galaxies $\mathrm{sr}^{-1}$ ). Values of $\sigma_{g}$ were increased in intervals of $10^{1 / 3}$ (beginning with $10^{2 / 3}$ ), which corresponds nominally to a volume density enhancement $\rho_{g}=10^{1 / 2}$ (beginning with 10).

In order to reduce the role of chance line-of-sight galaxy projections creating false clusters in the catalog, we sought an enhancement level low enough to select all the MKW, AWM, and WP clusters, but no lower. The two most optimum levels were found to be $\sigma_{g}=10^{4 / 3} \approx 21\left(\sigma_{21}\right)$ and $\sigma_{g}=10^{5 / 3} \approx 46\left(\sigma_{46}\right)$, corresponding to $\rho_{g}=100$ and $\rho_{g}=$ 316 , respectively. The $\sigma_{21}$ results were used to identify the clusters, and the $\sigma_{46}$ information was used both to detect regions of hierarchical subclustering and to identify a subsample of poor clusters at a higher density. Subsequent analysis has shown that projection effects play a negligible role in these poor clusters (Burns et al. 1996; Bhavsar 1980). The number of poor clusters detected at $\sigma_{21}$ and $\sigma_{46}$, along with the total number of galaxies that are members of these poor clusters, are listed in Table 1. The typical aperture radius of a three-member cluster at $\sigma_{21}$ is 0.146 ; at $\sigma_{46}$ it is 0.099 . To excellent approximation, for richer clusters, the aperture size scales with the square root of the number of members.

\section{THE POOR-CLUSTER CATALOG}

The following selection criteria were used to create the full poor-cluster catalog:

$$
\sigma_{g} \geq 10^{4 / 3}=\sigma_{21}, \quad m_{\mathrm{pg}} \leq 15.7, \quad N_{g} \geq 3,
$$

where $\sigma_{g}$ is the surface density of galaxies above the background, as defined in $\S 2, m_{\mathrm{pg}}$ is the photographic magnitude of each galaxy as recorded in the CGCG, and $N_{g}$ is the number of CGCG galaxies in each cluster. This catalog is presented in Table 2.

The electronic version of the CGCG we used has 15,409 galaxies within the Galactic caps. In this region, the WBL clusters contain 2245 galaxies at $\sigma_{21}$ and 1159 galaxies at $\sigma_{46}$. The total area of this region is $2.24431 \mathrm{sr}$. The area occupied by WBL clusters in this region is $0.01416 \mathrm{sr}$ at $\sigma_{21}$ and $0.00346 \mathrm{sr}$ at $\sigma_{46}$. Thus the WBL clusters contain about $14.6 \%$ of the galaxies in $0.63 \%$ of the area at $\sigma_{21}$ and $7.5 \%$ of the galaxies in $0.15 \%$ of the area at $\sigma_{46}$. These results make clear that this catalog contains poor clusters of high galaxy density.

The summary data for each poor cluster are presented in Table 2, and the data on individual galaxy members in Table 3. Both Tables 2 and 3 will be available in electronic form from the Astronomical Data Center. ${ }^{3}$ Table 4 contains a cross-reference to previously used names for these poor clusters. The new clusters are designated "WBL" to represent the last names of the authors, which is consistent with IAU guidelines.

\subsection{The Poor-Cluster Catalog: Table 2}

This table presents basic data on all 732 poor clusters. The following information is provided:

Column (1).-The WBL designation of each poor cluster. Cross-references to previously used names are given in Table 4. Footnotes in this column provide additional information for the poor clusters where necessary.

\footnotetext{
${ }^{3}$ http://adc.gsfc.nasa.gov/.
}

TABLE 1

Poor-Cluster Statistics at Different Surface Densities

\begin{tabular}{lrrrrrrrrrr}
\hline \hline Galaxy Surface Density & $N_{3}{ }^{\mathrm{a}}$ & $N_{4}{ }^{\mathrm{a}}$ & $N_{5}{ }^{\mathrm{a}}$ & $N_{6}{ }^{\mathrm{a}}$ & $N_{7}{ }^{\mathrm{a}}$ & $N_{8}{ }^{\mathrm{a}}$ & $N_{9}{ }^{\mathrm{a}}$ & $N_{>9}{ }^{\mathrm{a}}$ & $N_{\text {tot }}{ }^{\mathrm{b}}$ & $G_{\text {tot }}{ }^{\mathrm{c}}$ \\
\hline$\sigma_{21} \ldots \ldots \ldots \ldots \ldots \ldots \ldots \ldots \ldots \ldots \ldots \ldots \ldots \ldots \ldots \ldots \ldots$ & 446 & 127 & 54 & 31 & 25 & 10 & 9 & 30 & 732 & 3324 \\
$\sigma_{46} \ldots \ldots \ldots \ldots \ldots \ldots \ldots \ldots$ & 61 & 23 & 15 & 6 & 6 & 6 & 12 & 391 & 1691 \\
\hline
\end{tabular}

a $N_{i}=$ number of poor clusters containing $i$ Zwicky galaxies.

b Number of poor clusters with three or more Zwicky galaxies.

${ }^{c}$ Number of Zwicky galaxies in poor clusters. 


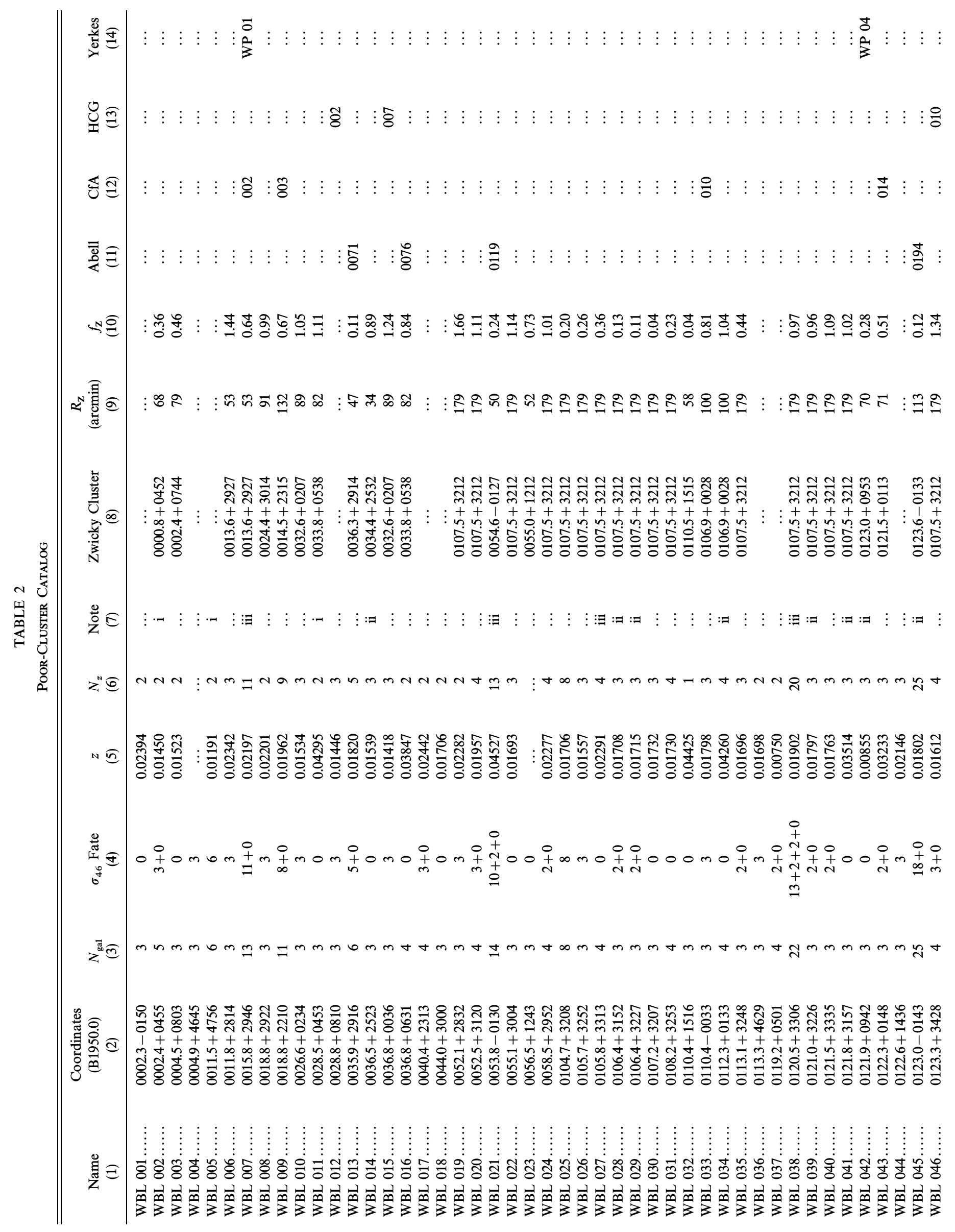




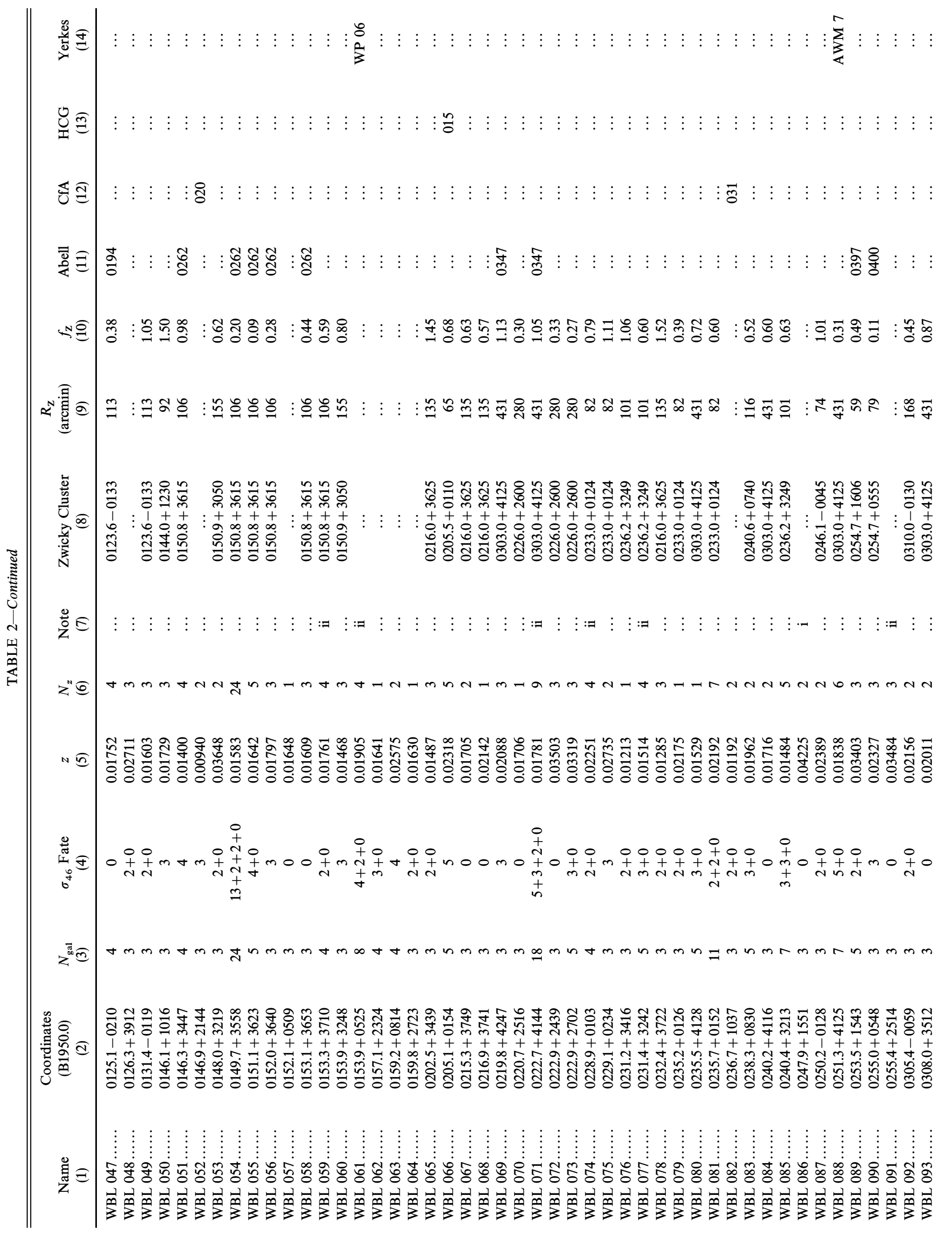




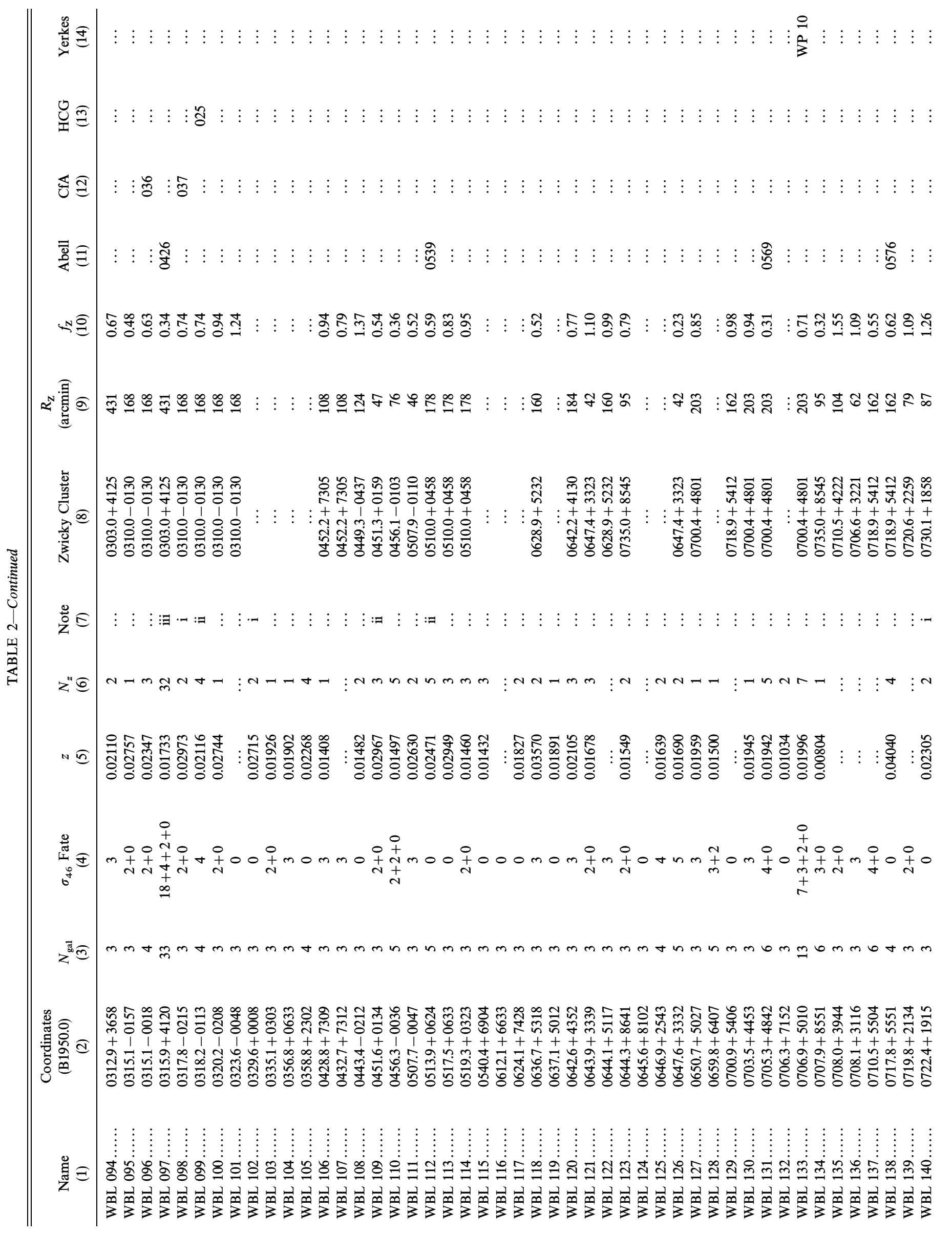




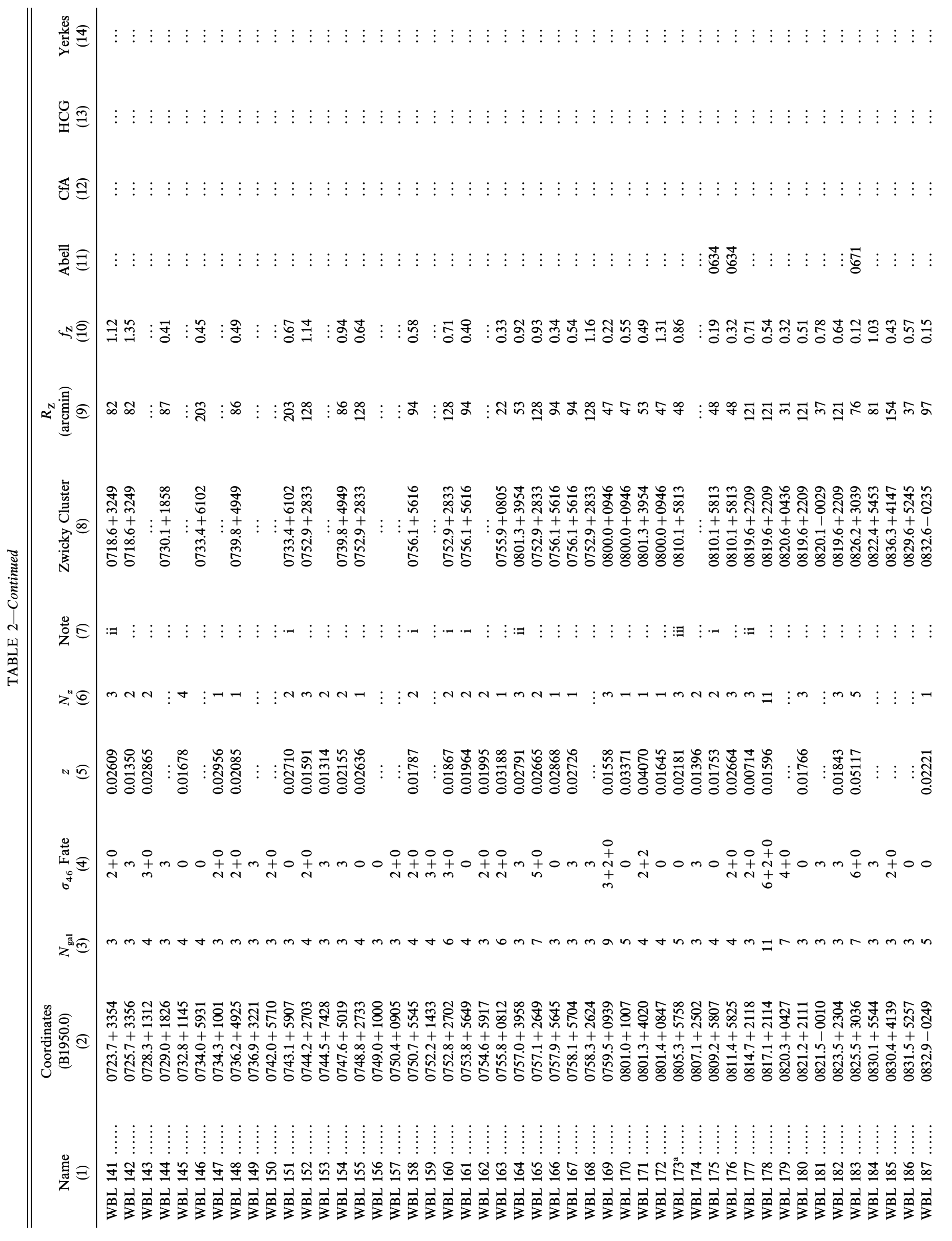




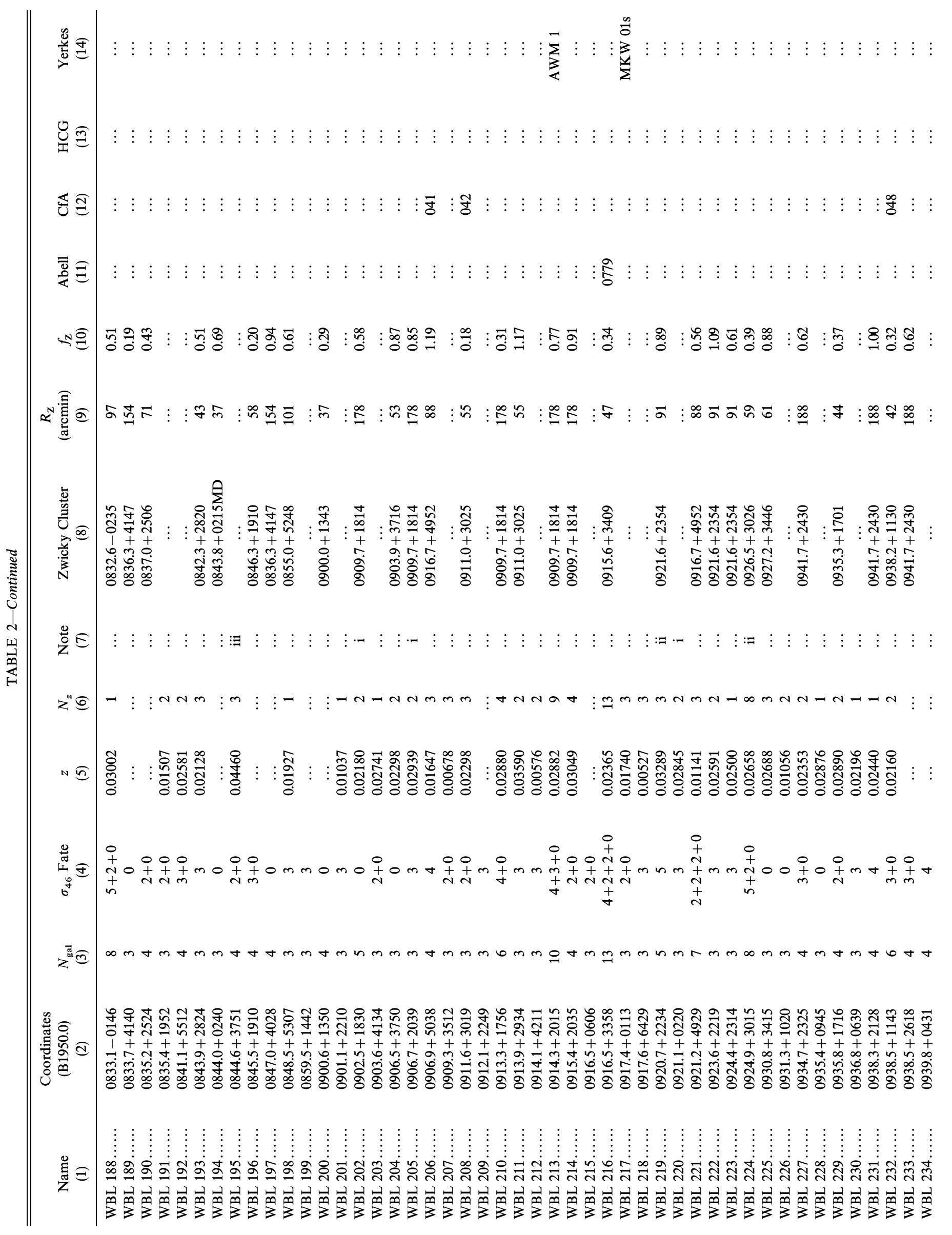




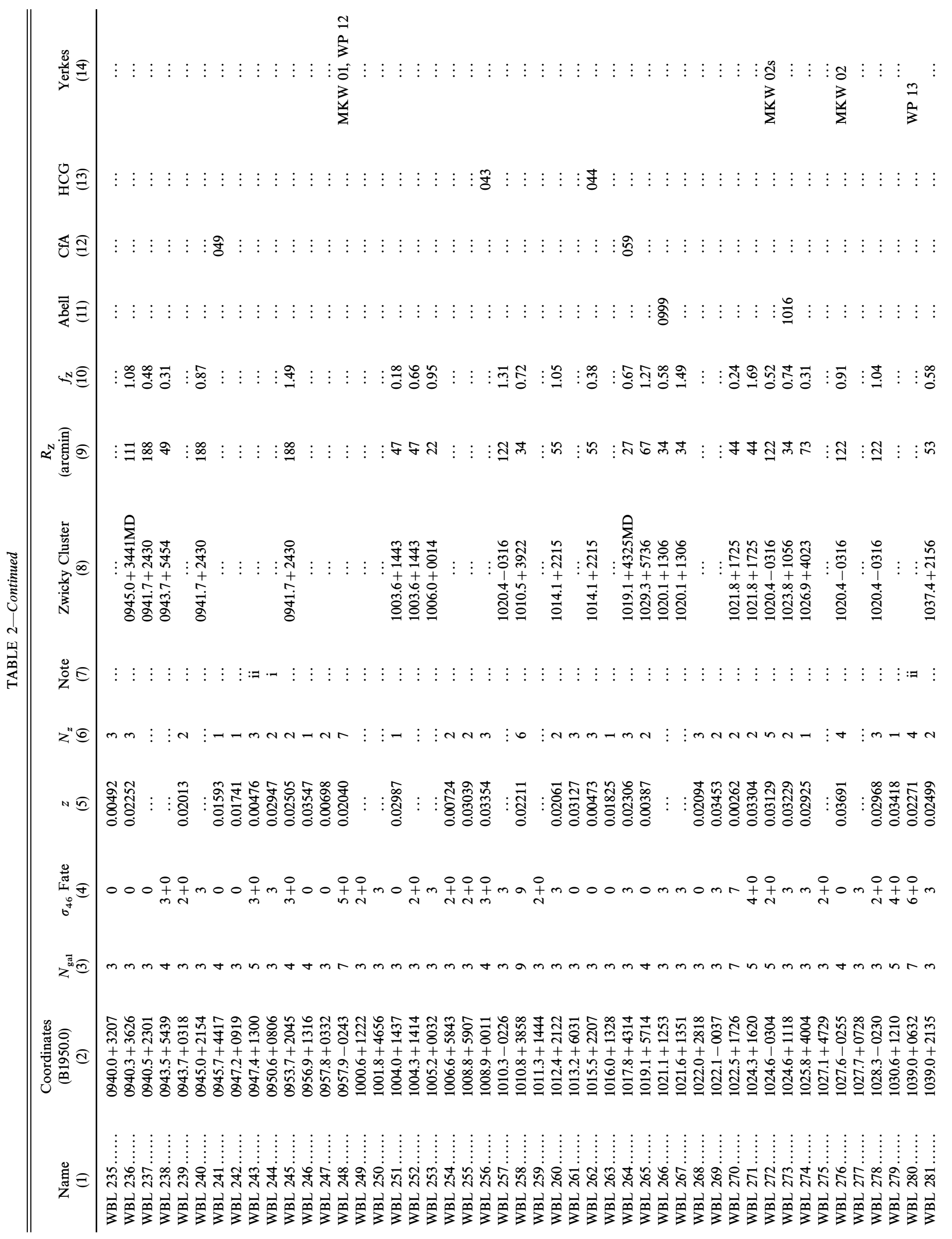




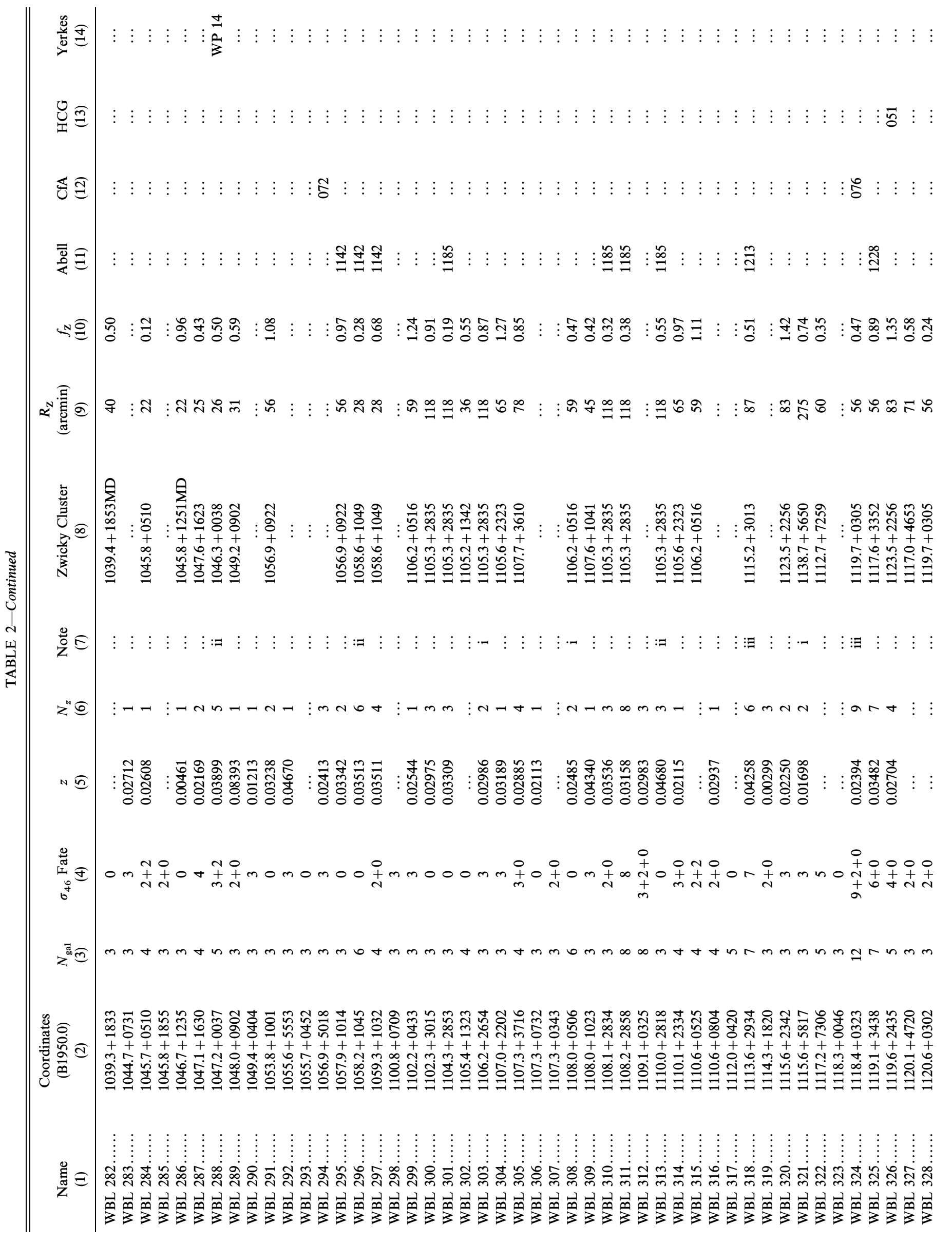




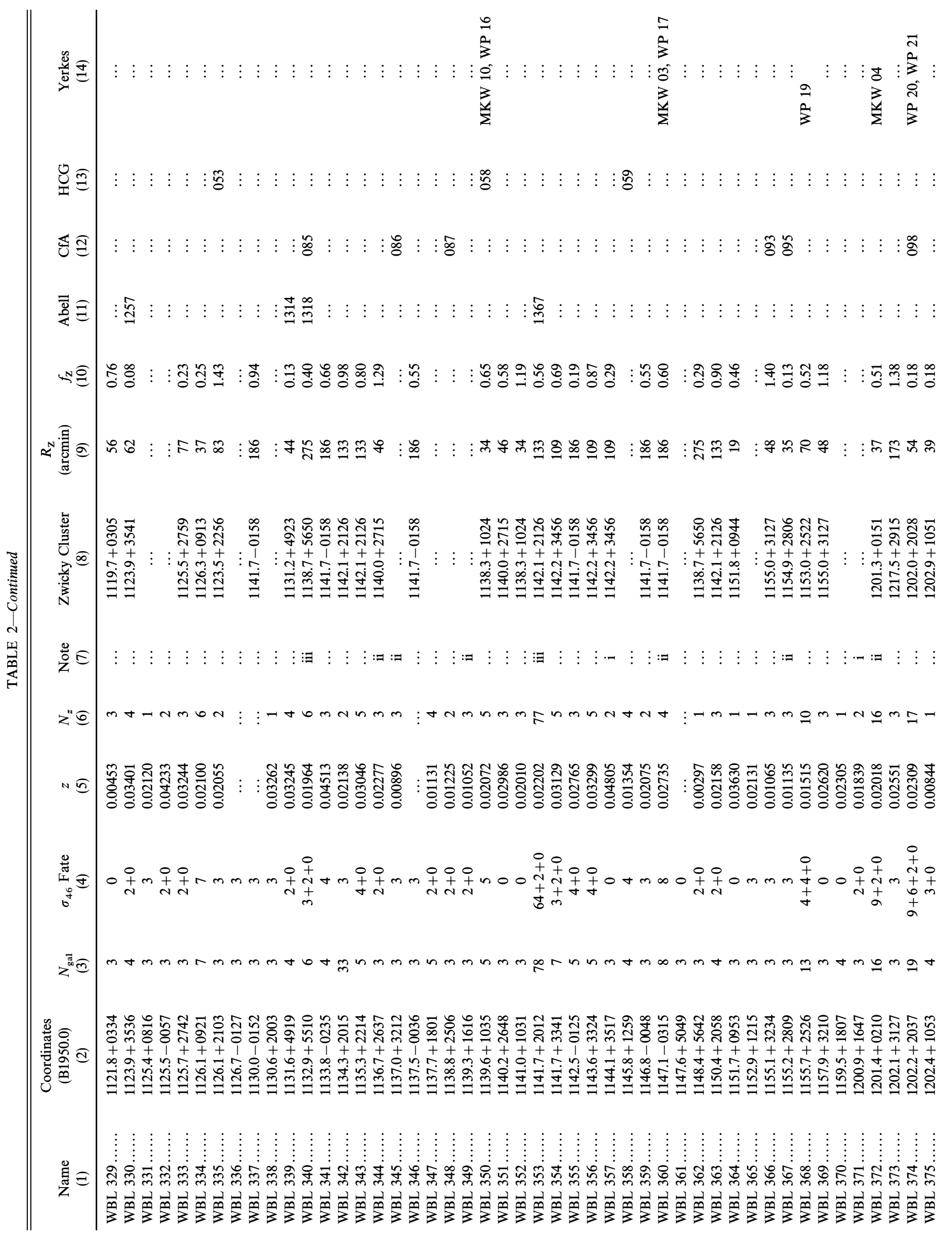




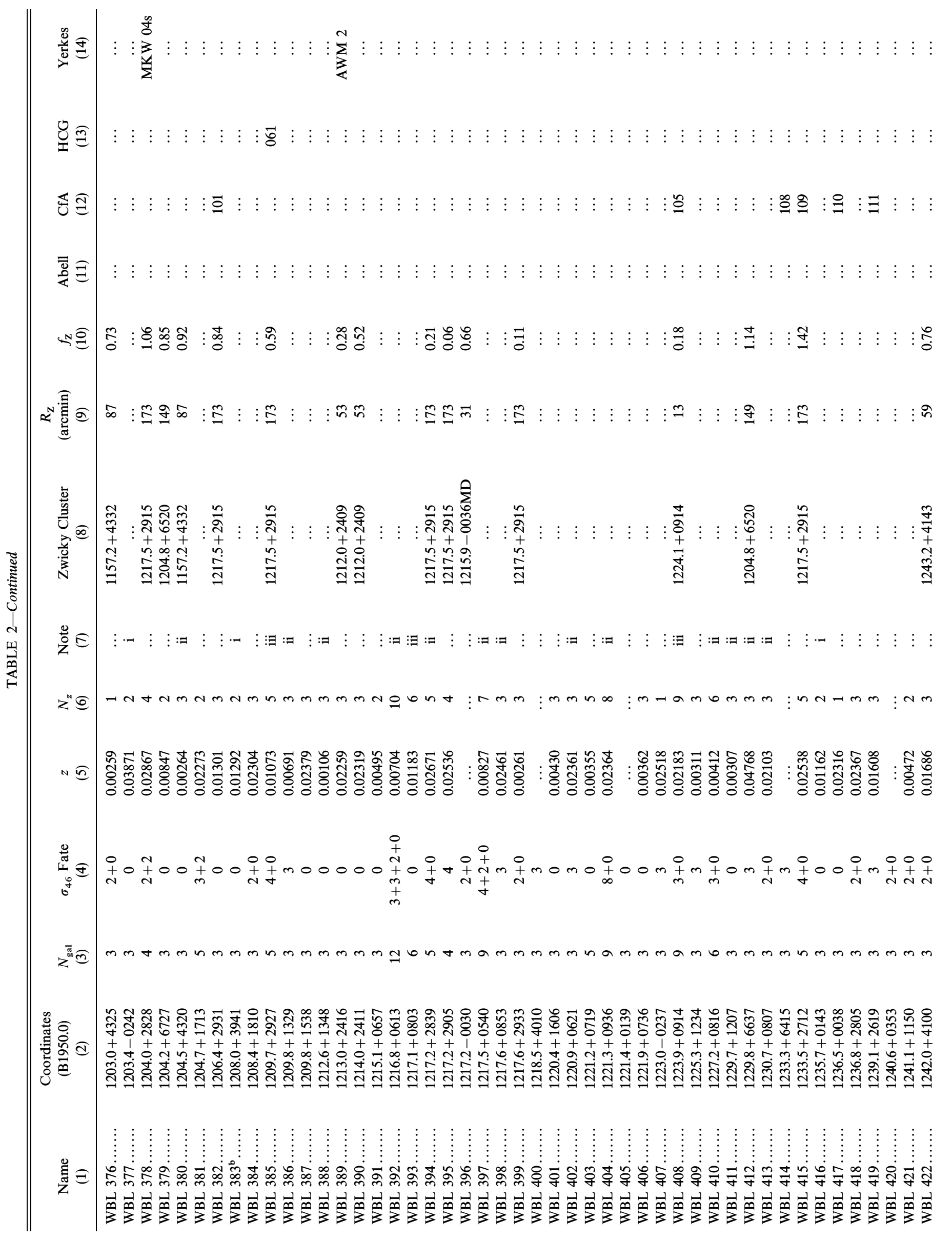




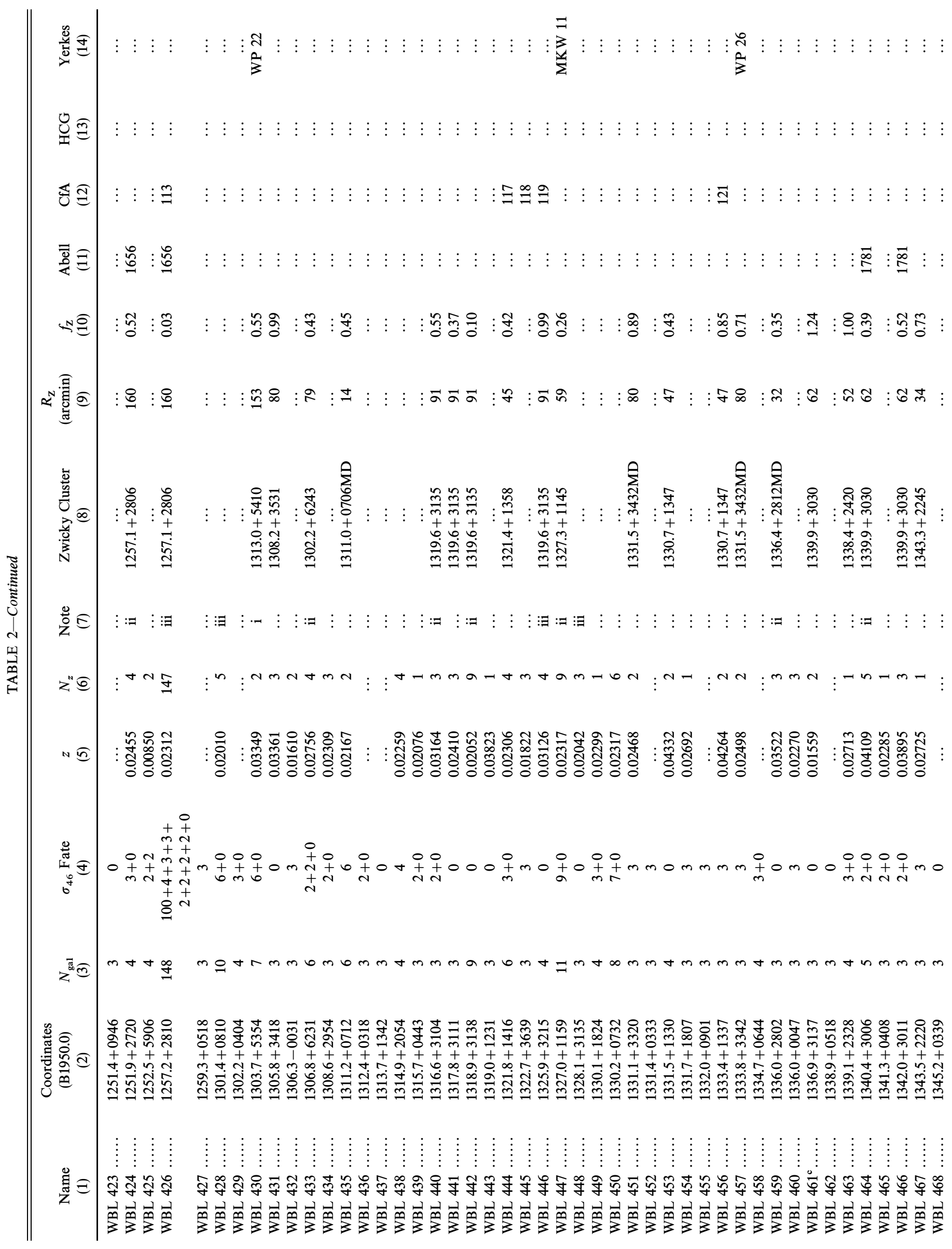




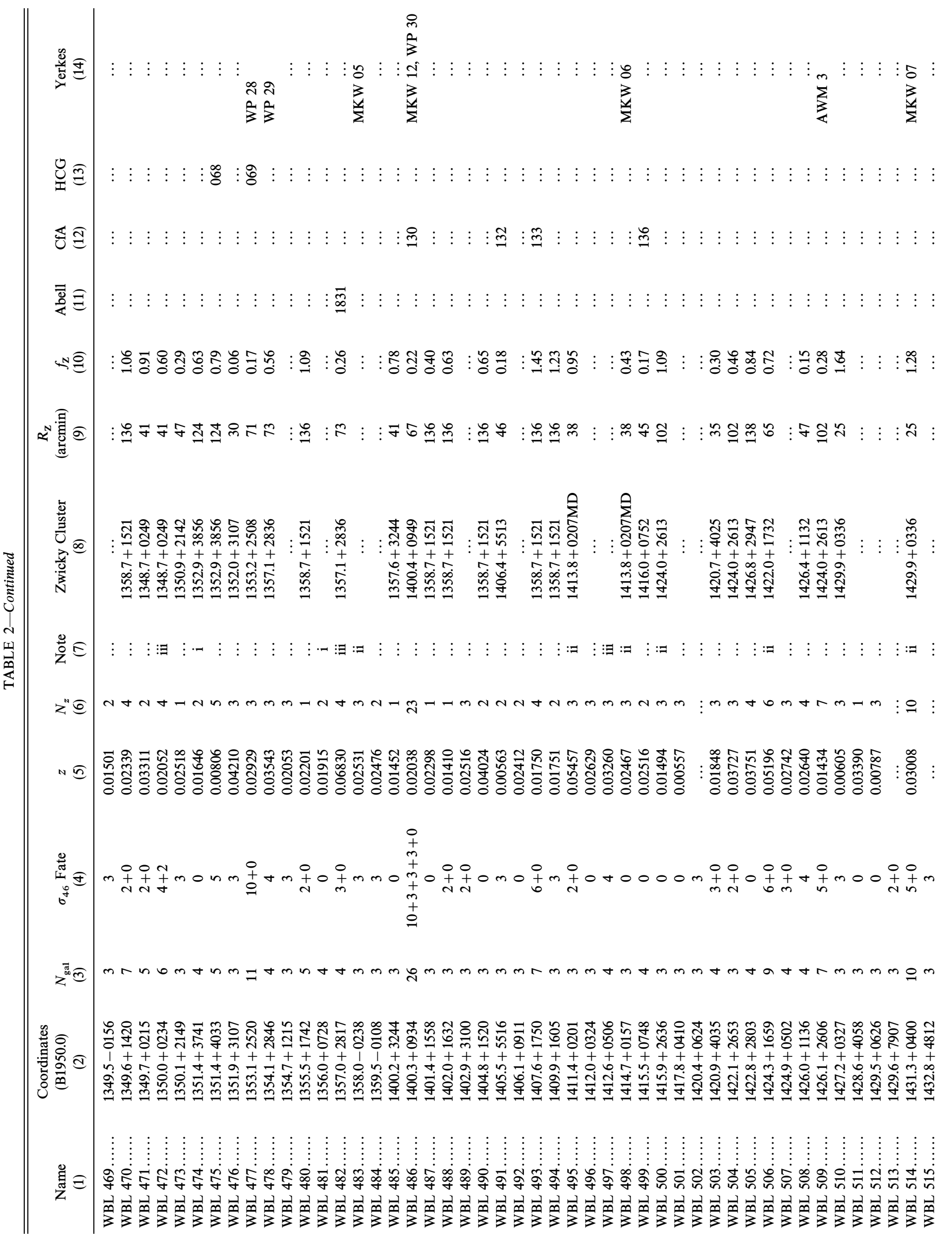




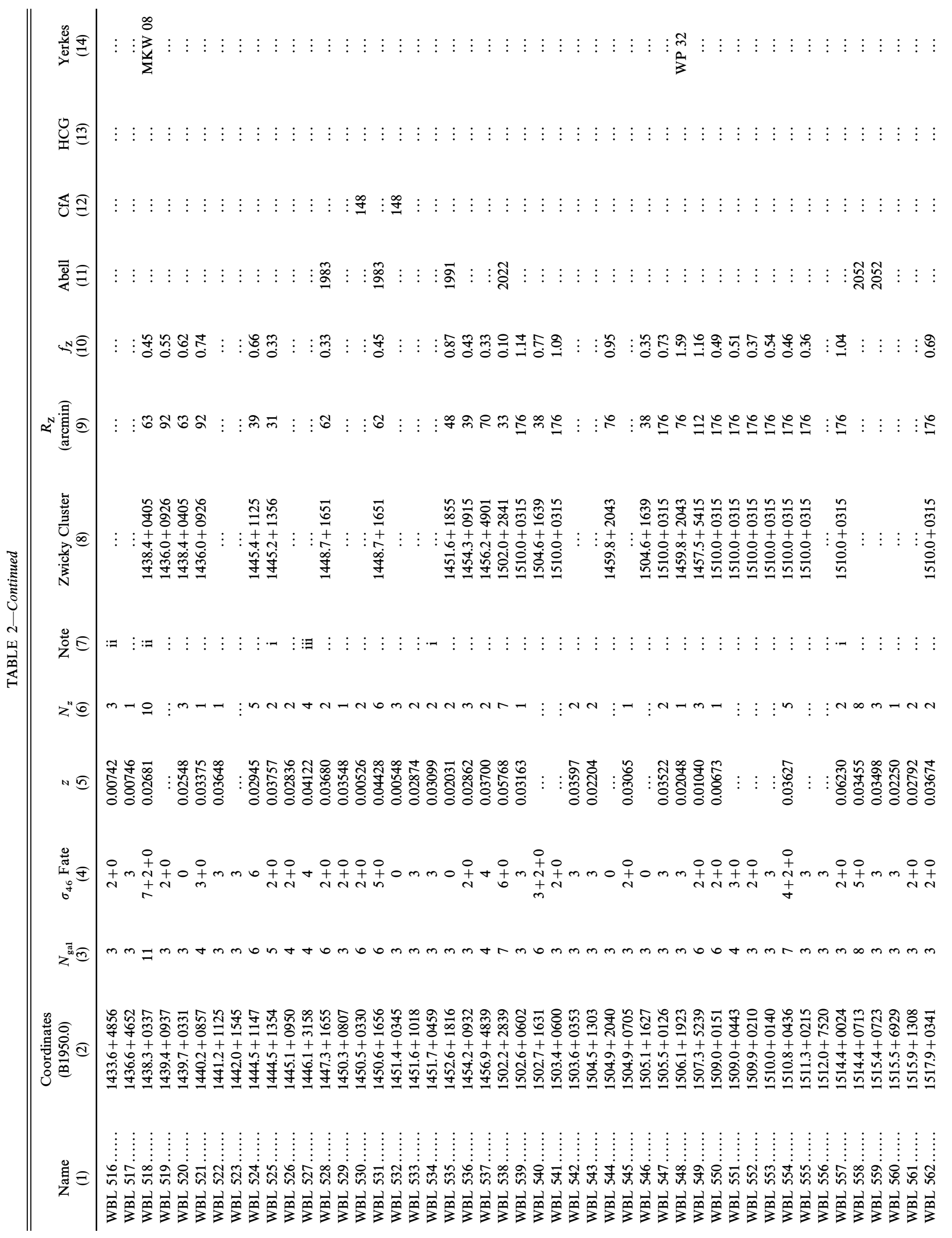




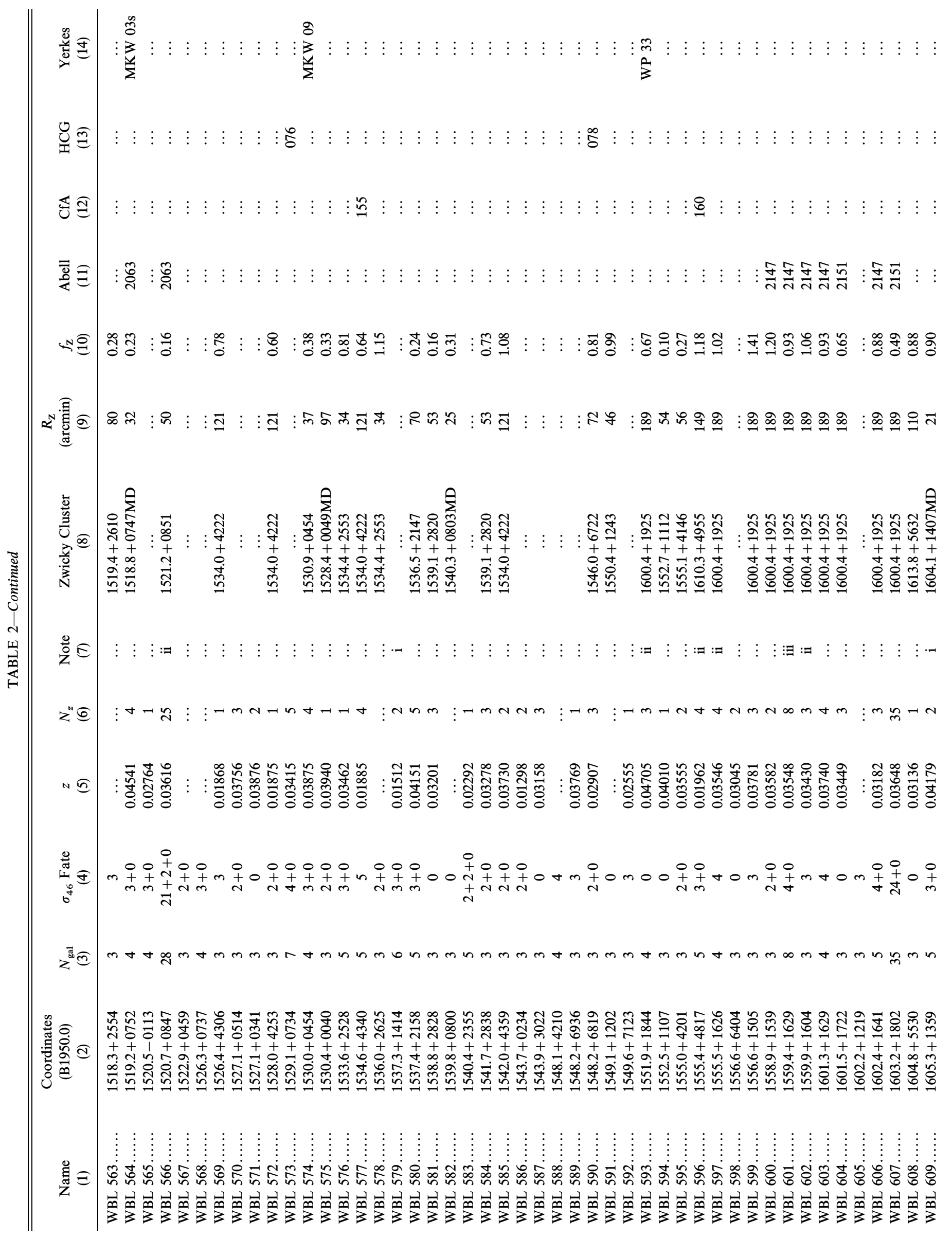




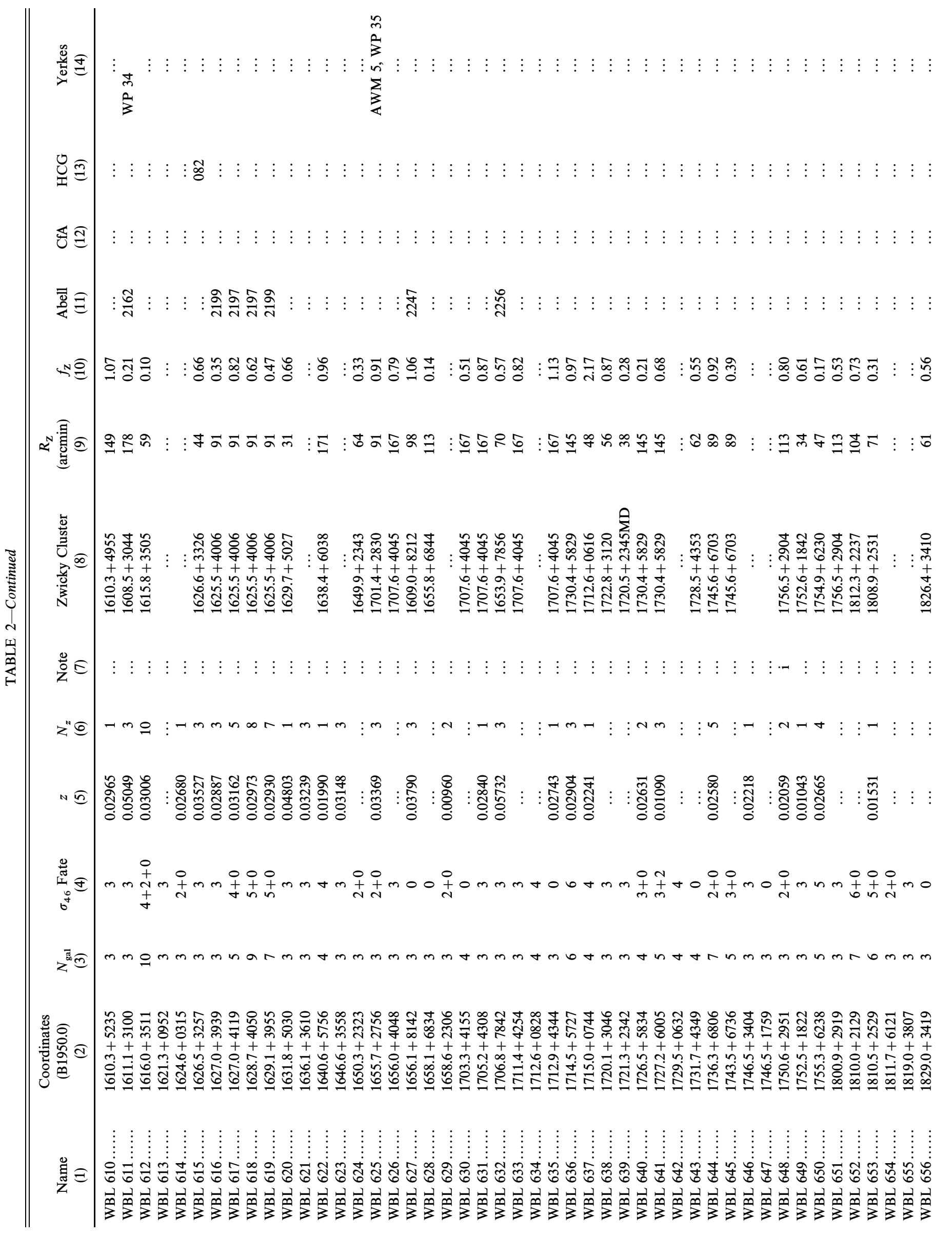




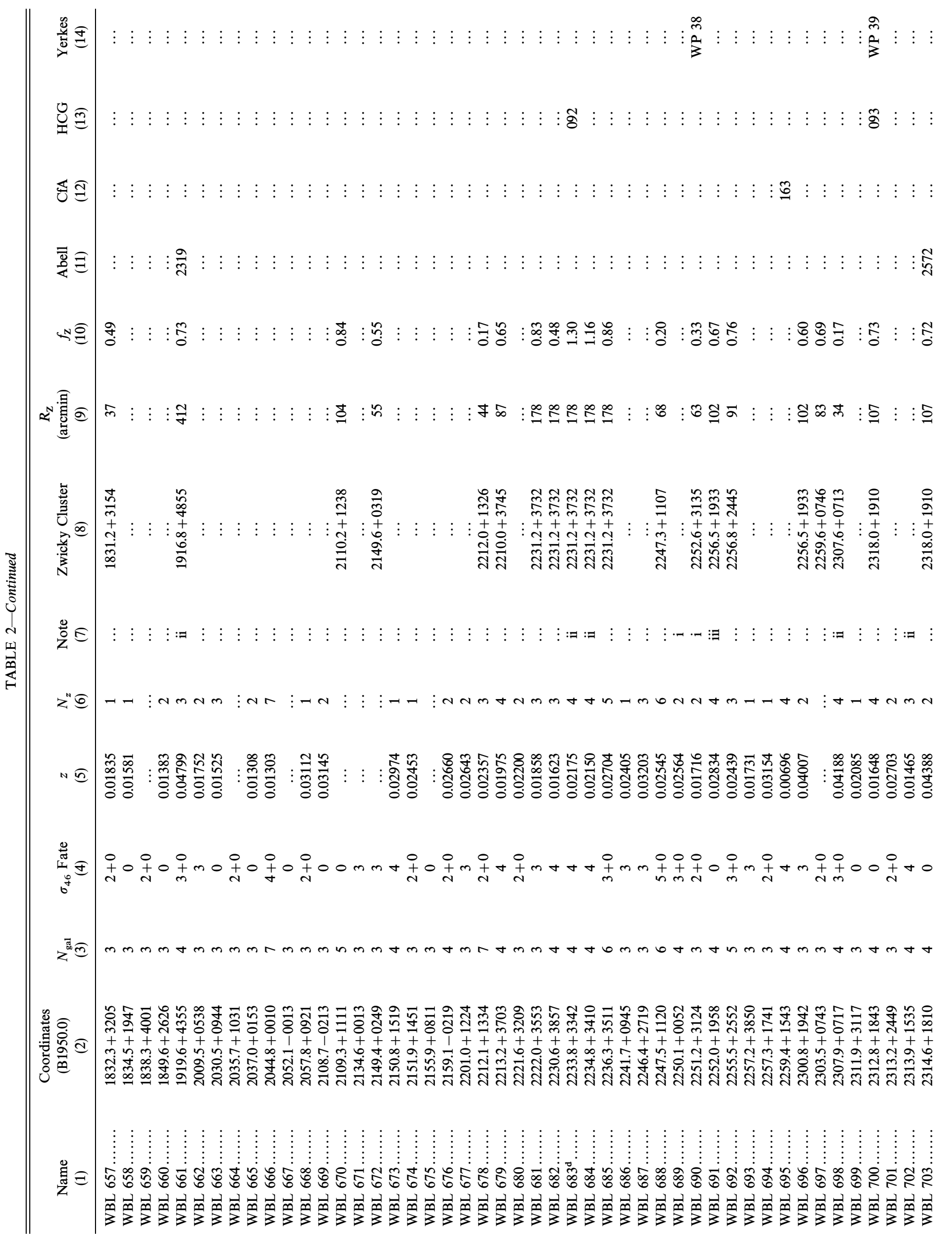




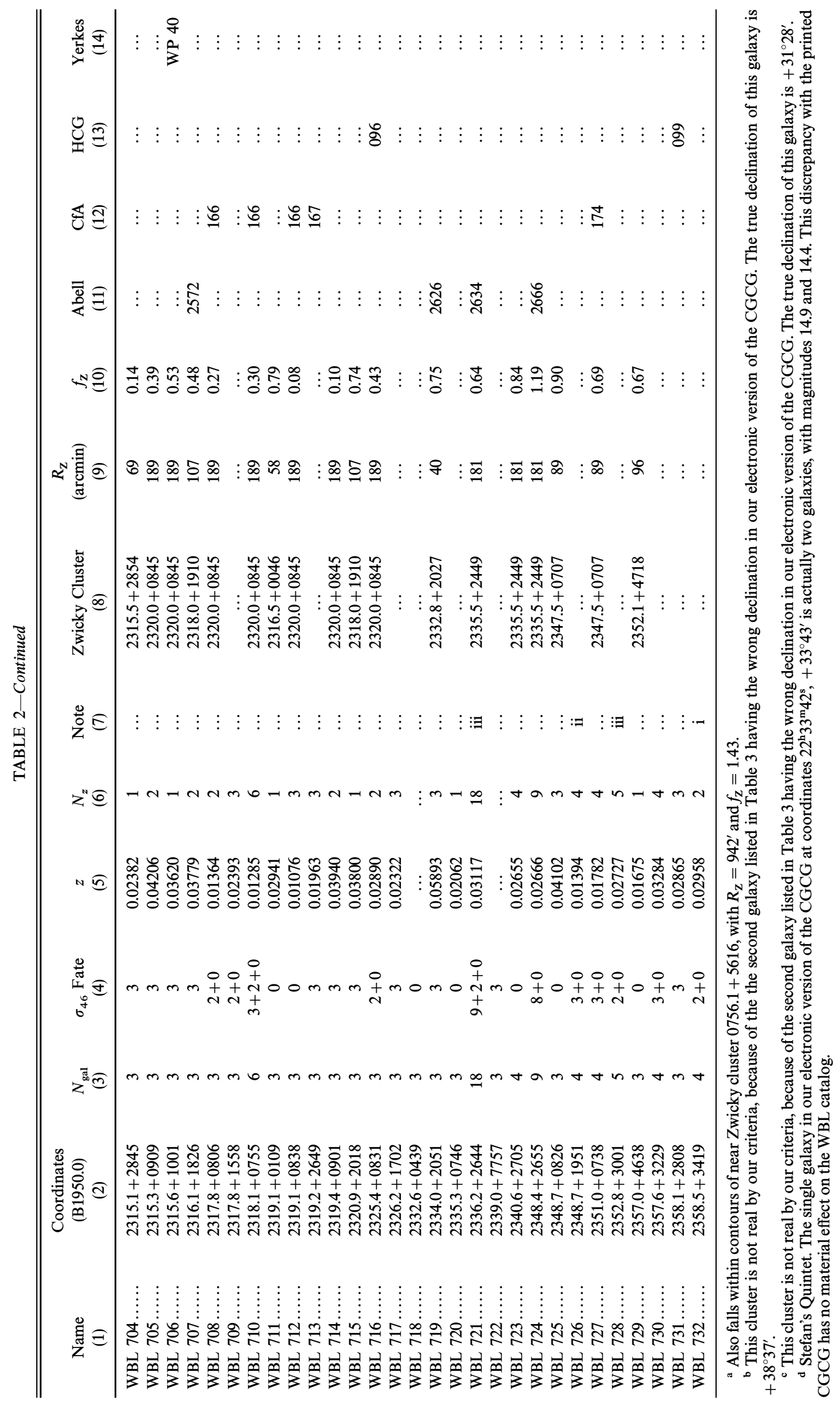


TABLE 3

POOR-Cluster Galaxies

\begin{tabular}{|c|c|c|c|c|c|c|c|c|}
\hline $\begin{array}{l}\text { Name } \\
\text { (1) }\end{array}$ & $\begin{array}{l}\text { R.A. (B1950.0) } \\
\text { (2) }\end{array}$ & $\begin{array}{c}\text { Decl. (B1950) } \\
\text { (3) }\end{array}$ & $\begin{array}{l}m_{\mathrm{pg}} \\
(4)\end{array}$ & $\begin{array}{l}N_{21} \\
(5)\end{array}$ & $\begin{array}{l}N_{46} \\
(6)\end{array}$ & $\begin{array}{c}\sigma_{46} \text { Fate } \\
\text { (7) }\end{array}$ & $\begin{array}{c}z \\
(8)\end{array}$ & $\begin{array}{l}\text { Other Names } \\
\text { (9) }\end{array}$ \\
\hline WBL $214-001 \ldots . .$. & 091506 & +2041 & 15.6 & 0 & 0 & $\ldots$ & 0.03031 & $\ldots$ \\
\hline WBL $214-002 \ldots \ldots$. & 091524 & +2035 & 15.7 & 3 & 1 & A & 0.03185 & $\ldots$ \\
\hline WBL $214-003 \ldots \ldots$. & 091530 & +2028 & 15.5 & 0 & 0 & $\ldots$ & 0.02788 & $\ldots$ \\
\hline WBL $214-004 \ldots \ldots$ & 091536 & +2037 & 15.7 & 1 & 1 & A & 0.03193 & $\ldots$ \\
\hline WBL $215-001 \ldots \ldots$ & 091618 & +0606 & 14.9 & 1 & 1 & A & $\ldots$ & $\ldots$ \\
\hline WBL $215-002 \ldots \ldots$. & 091630 & +0606 & 15.5 & 2 & 1 & A & $\ldots$ & $\ldots$ \\
\hline WBL $215-003 \ldots \ldots$ & 091654 & +0606 & 15.7 & 1 & 0 & $\ldots$ & $\ldots$ & $\ldots$ \\
\hline WBL $216-001 \ldots \ldots$ & 091518 & +3346 & 15.6 & 0 & 0 & $\ldots$ & 0.02401 & $\ldots$ \\
\hline WBL $216-002 \ldots .$. & 091518 & +3404 & 15.6 & 1 & 1 & A & 0.02220 & $\ldots$ \\
\hline WBL $216-003 \ldots . .$. & 091530 & +3404 & 15.6 & 1 & 1 & A & 0.02414 & $\ldots$ \\
\hline WBL $216-004 \ldots . .$. & 091612 & +3404 & 15.6 & 10 & 1 & B & 0.02341 & NGC 2827, IC 2460 \\
\hline WBL $216-005 \ldots . .$. & 091612 & +3413 & 15.7 & 0 & 0 & $\ldots$ & 0.02366 & NGC 2823, UGC 04935 \\
\hline WBL $216-006 \ldots . .$. & 091618 & +3357 & 15.3 & 12 & 0 & $\dddot{\mathrm{C}}$ & 0.02659 & NGC 2825 \\
\hline WBL $216-007 \ldots . .$. & 091624 & +3350 & 14.6 & 0 & 0 & $\ldots$ & 0.02473 & NGC 2826, UGC 04939 \\
\hline WBL $216-008 \ldots \ldots$. & 091630 & +3405 & 15.7 & 10 & 1 & B & 0.02166 & NGC 2828 \\
\hline WBL $216-009 . . . .$. & 091642 & +3357 & 15.4 & 8 & 3 & $\mathrm{C}$ & 0.02037 & NGC 2830, UGC 04941 \\
\hline WBL $216-010 \ldots \ldots$. & 091648 & +3358 & 13.3 & 8 & 2 & $\mathrm{C}$ & 0.02096 & NGC 2832, UGC 04942 \\
\hline WBL $216-011 \ldots \ldots$. & 091654 & +3408 & 15.6 & 1 & 0 & $\ldots$ & 0.02475 & NGC 2833 \\
\hline WBL $216-012 \ldots . .$. & 091700 & +3355 & 15.6 & 8 & 2 & $\mathrm{C}$ & 0.02351 & NGC 2834 \\
\hline WBL $216-013 \ldots .$. & 091736 & +3352 & 15.3 & 0 & 0 & $\ldots$ & 0.02743 & NGC 2839 \\
\hline WBL $217-001 \ldots \ldots$. & 091718 & +0109 & 15.4 & 2 & 1 & A & 0.01778 & $\ldots$ \\
\hline WBL $217-002 \ldots \ldots$. & 091724 & +0108 & 15.5 & 2 & 1 & A & 0.01724 & $\ldots$ \\
\hline WBL $217-003 \ldots . .$. & 091730 & +0115 & 14.1 & 0 & 0 & $\ldots$ & 0.01718 & UGC 04956 \\
\hline WBL $218-001 \ldots \ldots$ & 091706 & +6428 & 14.0 & 2 & 2 & A & 0.00544 & NGC 2814 \\
\hline WBL $218-002 \ldots \ldots$ & 091730 & +6427 & 15.1 & 2 & 2 & $\mathrm{~A}$ & 0.00512 & NGC 2820 , IC 2458 \\
\hline WBL $218-003 \ldots . .$. & 091748 & +6429 & 13.1 & 2 & 2 & A & 0.00525 & NGC 2820, UGC 04961 \\
\hline WBL $219-001 \ldots . .$. & 092030 & +2232 & 15.2 & 4 & 2 & A & 0.00049 & UGC 04991 \\
\hline WBL $219-002 \ldots . .$. & 092030 & +2240 & 15.7 & 0 & 0 & A & $\ldots$ & IC 2465 \\
\hline WBL 219-003...... & 092036 & +2233 & 15.1 & 4 & 2 & A & 0.03437 & UGC 04991 \\
\hline WBL $219-004 \ldots \ldots$. & 092048 & +2234 & 15.7 & 4 & 4 & A & $\ldots$ & $\ldots$ \\
\hline WBL $219-005 \ldots . .$. & 092112 & +2233 & 15.5 & 1 & 0 & A & 0.03141 & $\ldots$ \\
\hline
\end{tabular}

NoтE.-Table 3 is presented in its entirety in the electronic edition of the Astronomical Journal. A portion is shown here for guidance regarding its form and content. Units of right ascension are hours, minutes, and seconds, and units of declination are degrees and arcminutes.

Column (2).-Coordinates. The right ascension and declination, equinox B1950.0, for the centroid of the poor cluster are listed. The centroid is determined from a luminosity-weighted mean of all member galaxies listed in the CGCG. Luminosities were computed as $L=10^{-0.4 m_{\mathrm{pg}}}$ and used as the weight in computing the mean right ascension and declination for each cluster, i.e., $\langle$ R.A. $\rangle=$ $\sum_{i}(\text { R.A. })_{i} L_{i} / \sum_{i} L_{i}$

Column (3).- Richness. The number of CGCG galaxies in each poor cluster.

Column (4).-Clustering at $\sigma_{46}$. An indication of the fate of each individual galaxy at the higher density enhancement $\left(\sigma_{46}\right)$. A single zero in this column indicates that a group at $\sigma_{21}$ fractured into single galaxies at $\sigma_{46}$ (i.e., the galaxy apertures did not overlap). Combinations of other numbers indicate how many galaxies were in each subcluster at $\sigma_{46}$, with " 0 " indicating one or more isolated galaxies. For example, a cluster with 11 members and an $8+0$ in this column becomes a group of eight members with three isolated galaxies at $\sigma_{46}$. A cluster of 11 members and an entry of $4+3+2+0$ breaks up into three subclusters of four, three, and two members, with two isolated galaxies at $\sigma_{46}$.
Columns (5)-(7).--Poor-cluster redshift. Column (5) lists a redshift for the cluster, when available, computed as an average of redshifts from the literature obtained through the NASA Extragalactic Database (NED). ${ }^{4}$ Column (6) $\left(N_{z}\right)$ indicates the number of galaxy redshifts available from NED. Column (7) ("Note") gives notes on the determination of the cluster redshift. If there is no note in column (7), the number of galaxy redshifts listed in column (6) were used to compute the cluster redshift in column (5). If the note is (i), there were only two redshifts available and their values differed by more than $1500 \mathrm{~km} \mathrm{~s}^{-1}$. In this case, the two galaxies may be close only in projection. The redshift given in this case is the average of the two galaxy redshifts. A note of (ii) indicates that, of the three or more redshifts available, one was more than $1500 \mathrm{~km} \mathrm{~s}^{-1}$ from the mean. After removing this discrepant redshift, a new mean was calculated, with all remaining redshifts within $1500 \mathrm{~km} \mathrm{~s}^{-1}$

${ }^{4}$ The NASA/IPAC Extragalactic Database is operated by the Jet Propulsion Laboratory, California Institute of Technology, under contract with the National Aeronautics and Space Administration. 
TABLE 4

Old Poor-Cluster Designations Used in the Literature

\begin{tabular}{|c|c|c|c|c|c|c|c|}
\hline Old Name & New Name & Old Name & New Name & Old Name & New Name & Old Name & New Name \\
\hline AWM 1 & WBL 213 & WP 33. & WBL 593 & N56-296...... & WBL 298 & N79-238. & WBL 419 \\
\hline AWM $2 \ldots \ldots$. & WBL 389 & WP $34 \ldots \ldots$. & WBL 611 & N56-297...... & WBL 299 & N79-266 ....... & WBL 375 \\
\hline AWM $3 \ldots \ldots$. & WBL 509 & WP $35 \ldots \ldots$ & WBL 625 & N56-306...... & WBL 336 & N79-268 ....... & WBL 395 \\
\hline AWM $4 \ldots \ldots .$. & $\mathrm{a}, \mathrm{b}$ & WP $36 \ldots \ldots$ & c & N56-308 ...... & WBL 346 & N79-270. & WBL 438 \\
\hline AWM $5 \ldots . . .$. & WBL 625 & WP $37 \ldots \ldots$. & c & N56-329...... & WBL 522 & N79-276 . & WBL 478 \\
\hline AWM $6 \ldots \ldots .$. & b & WP $38 \ldots \ldots$ & WBL 690 & N56-332 ..... & WBL 533 & N79-278 & WBL 343 \\
\hline AWM $7 \ldots \ldots \ldots$ & WBL 088 & WP $39 \ldots \ldots$ & WBL 700 & N56-336...... & WBL 539 & N79-280 & WBL 356 \\
\hline MKW $01 \ldots . .$. & WBL 248 & WP $40 \ldots \ldots$ & WBL 706 & N56-338..... & WBL 542 & N79-281 ....... & WBL 381 \\
\hline MKW $02 \ldots \ldots$ & WBL 276 & WP $41 \ldots \ldots$. & b & N56-339..... & WBL 543 & N79-282 . & WBL 385 \\
\hline MKW $03 \ldots . . .$. & WBL 360 & WP $42 \ldots \ldots$ & c & N56-343 ..... & WBL 548 & N79-283 & WBL 394 \\
\hline MKW $04 \ldots \ldots$. & WBL 372 & K103 .......... & WBL 312 & N56-355...... & WBL 287 & N79-284 . & WBL 415 \\
\hline MKW $05 \ldots . . .$. & WBL 483 & K131 ......... & WBL 472 & N56-359..... & WBL 341 & N79-286 ....... & WBL 475 \\
\hline MKW $06 \ldots . . .$. & WBL 498 & K140 .......... & WBL 499 & N56-361 ...... & WBL 497 & N79-290 ....... & WBL 354 \\
\hline MKW $07 \ldots \ldots$. & WBL 514 & K179 .......... & WBL 226 & N56-365 ...... & WBL 537 & N79-292 ...... & WBL 404 \\
\hline MKW $08 \ldots . . . .$. & WBL 518 & $\mathrm{~K} 30 \ldots \ldots \ldots$ & WBL 288 & N56-366.......... & WBL 588 & N79-293 ............. & WBL 408 \\
\hline MKW $09 . . . . .$. & WBL 574 & K315 .......... & WBL 438 & N56-367...... & WBL 271 & N79-295 ....... & WBL 428 \\
\hline MKW $10 \ldots \ldots$ & WBL 350 & K36 .......... & WBL 323 & N56-368 ...... & WBL 279 & N79-296 ....... & WBL 447 \\
\hline MKW $11 \ldots . .$. & WBL 447 & N34-169 ...... & WBL 210 & N56-369..... & WBL 288 & N79-297 ....... & WBL 477 \\
\hline MKW $12 \ldots \ldots$ & WBL 486 & N34-170........ & WBL 234 & N56-371 ........ & WBL 355 & N79-298a ........ & WBL 368 \\
\hline MKW 01s...... & WBL 217 & N34-171 ..... & WBL 622 & N56-373..... & WBL 576 & N79-298b ...... & WBL 368 \\
\hline MKW $02 s . . . .$. & WBL 272 & N34-172 ...... & WBL 248 & N56-374 ..... & WBL 577 & N79-299a ...... & WBL 374 \\
\hline MKW 03s...... & WBL 564 & N34-173 ...... & WBL 650 & N56-381 ..... & WBL 524 & N79-299b ...... & WBL 374 \\
\hline MKW 04s...... & WBL 378 & N34-175 ..... & WBL 636 & N56-385 ...... & WBL 540 & S34-087 ........ & WBL 008 \\
\hline WP $01 \ldots \ldots \ldots$ & WBL 007 & N45-273 ...... & WBL 205 & N56-387..... & WBL 270 & S34-088 ........ & WBL 017 \\
\hline WP $02 \ldots \ldots \ldots$ & c & N45-280...... & WBL 222 & N56-388 ...... & WBL 280 & S34-090 ........ & WBL 020 \\
\hline WP $03 \ldots$ & c & $\mathrm{N} 45-281 \ldots \ldots$ & WBL 223 & N56-391 .......... & WBL 312 & S34-091 …........... & WBL 026 \\
\hline WP $04 \ldots \ldots \ldots$ & WBL 042 & N45-285 ...... & WBL 230 & N56-392 ...... & WBL 360 & S34-093 ........ & WBL 062 \\
\hline WP $05 \ldots \ldots \ldots$ & c & N45-300...... & WBL 257 & N56-393...... & WBL 258 & S34-103 ........ & WBL 713 \\
\hline WP $06 \ldots \ldots \ldots$ & WBL 061 & N45-302 ...... & WBL 269 & N56-394 ...... & WBL 514 & S34-106 ........ & WBL 731 \\
\hline WP $07 \ldots \ldots \ldots$ & $\mathrm{c}$ & N45-309..... & WBL 550 & N56-395 ...... & WBL 518 & S34-110 ......... & WBL 009 \\
\hline WP $08 \ldots \ldots \ldots$ & c & $\mathrm{N} 45-342 \ldots \ldots$ & WBL 206 & N56-396...... & WBL 324 & S34-111 ........ & WBL 025 \\
\hline WP $09 \ldots \ldots \ldots$ & c & N45-346...... & WBL 231 & N67-238 ...... & WBL 419 & S34-112 ............ & WBL 724 \\
\hline WP $10 \ldots \ldots \ldots$ & WBL 133 & N45-348 ...... & WBL 234 & N67-244...... & WBL 303 & S34-113 ........ & WBL 038 \\
\hline WP $11 \ldots \ldots \ldots$ & $\mathrm{c}$ & N45-351 ...... & WBL 245 & N67-245 ...... & WBL 304 & S34-115 ........ & WBL 007 \\
\hline WP $12 \ldots \ldots \ldots$ & WBL 248 & N45-353 ...... & WBL 256 & N67-300 ...... & WBL 358 & S49-122 ........ & WBL 015 \\
\hline WP $13 \ldots \ldots \ldots$ & WBL 280 & N45-356...... & WBL 551 & N67-309...... & WBL 508 & S49-123 ......... & WBL 017 \\
\hline WP $14 \ldots \ldots \ldots$ & WBL 288 & N45-358 ...... & WBL 565 & N67-310...... & WBL 527 & S49-125 ........ & WBL 044 \\
\hline WP $15 \ldots \ldots \ldots$ & $\mathrm{c}$ & N45-360 ...... & WBL 574 & N67-311 ...... & WBL 326 & S49-126 ........ & WBL 050 \\
\hline WP $16 \ldots \ldots \ldots$ & WBL 350 & N45-361 ......... & WBL 588 & N67-312 ......... & WBL 350 & S49-127 ............. & WBL 075 \\
\hline WP $17 \ldots \ldots \ldots$ & WBL 360 & N45-363 ...... & WBL 597 & N67-317 ...... & WBL 435 & S49-128 ... & WBL 083 \\
\hline WP $18 \ldots \ldots \ldots \ldots$ & b & N45-365........ & WBL 622 & N67-318........ & WBL 472 & S49-130.. & WBL 686 \\
\hline WP $19 \ldots \ldots \ldots$ & WBL 368 & N45-366...... & WBL 219 & N67-320...... & WBL 318 & S49-132.. & WBL 698 \\
\hline WP $20 \ldots \ldots \ldots$ & WBL 374 & N45-367 ...... & WBL 243 & N67-321 ....... & WBL 325 & S49-134 ............ & WBL 706 \\
\hline WP $21 \ldots \ldots \ldots$ & WBL 374 & N45-368 ...... & WBL 248 & N67-322 ....... & WBL 334 & S49-137 ............. & WBL 717 \\
\hline WP $22 \ldots \ldots \ldots$ & WBL 430 & N45-371 ...... & WBL 322 & N67-323..... & WBL 430 & S49-138 ........ & WBL 726 \\
\hline WP $23 \ldots \ldots \ldots$ & c & N45-376...... & WBL 210 & N67-325 ...... & WBL 493 & S49-139 ........ & WBL 727 \\
\hline WP $24 \ldots \ldots \ldots$ & c & N45-377 ...... & WBL 232 & N67-326..... & WBL 509 & S49-140 ........ & WBL 061 \\
\hline WP $25 \ldots \ldots \ldots$ & b & N45-379...... & WBL 579 & N67-328...... & WBL 311 & S49-141 ......... & WBL 063 \\
\hline WP $26 \ldots \ldots \ldots$ & WBL 457 & N45-381 ...... & WBL 554 & N67-329...... & WBL 450 & S49-142 ........ & WBL 099 \\
\hline WP $27 \ldots \ldots \ldots$ & b & $\mathrm{N} 45-382 \ldots \ldots$ & WBL 573 & N67-330 ........... & WBL 397 & S49-143 .............. & WBL 695 \\
\hline WP $28 \ldots \ldots \ldots$ & WBL 477 & $\mathrm{~N} 45-383 \ldots \ldots$ & WBL 619 & N67-333 ......... & WBL 428 & S49-144 ............... & WBL 702 \\
\hline WP $29 \ldots \ldots \ldots$ & WBL 478 & N45-384 ...... & WBL 224 & N67-335...... & WBL 372 & S49-145 ........ & WBL 066 \\
\hline WP $30 \ldots \ldots \ldots$ & WBL 486 & N45-388 ...... & WBL 213 & N67-336...... & WBL 486 & S49-146 ........ & WBL 688 \\
\hline WP $31 \ldots \ldots \ldots$ & b & N45-389....... & WBL 612 & N79-207........ & WBL 335 & S49-147 ......... & WBL 009 \\
\hline WP $32 \ldots \ldots \ldots$ & WBL 548 & $\mathrm{~N} 45-391 \ldots \ldots$ & WBL 566 & N79-220....... & WBL 373 & & \\
\hline
\end{tabular}

${ }^{\text {a }}$ Cluster galaxies were too faint to be included in the WBL catalog.

${ }^{b}$ Cluster is too diffuse to meet the galaxy density criterion of the WBL catalog.

${ }^{c}$ Cluster is south of $-3^{\circ}$ declination and therefore not included in the WBL catalog.

from this new mean. A note of (iii) indicates that, of the three or more redshifts available, at least two discrepant redshifts were present. The redshift listed in column (7) is then the mean redshift calculated after removing only the most discrepant velocity. A value of $1500 \mathrm{~km} \mathrm{~s}^{-1}$ was chosen for the cutoff velocity because a "typical" poor cluster has a velocity dispersion, $\sigma_{v}$, of $\approx 500 \mathrm{~km} \mathrm{~s}^{-1}$ (Ledlow et al. 1996; Beers et al. 1995). This criteria is thus analogous to the standard $3 \sigma$ clipping often used in the literature. 


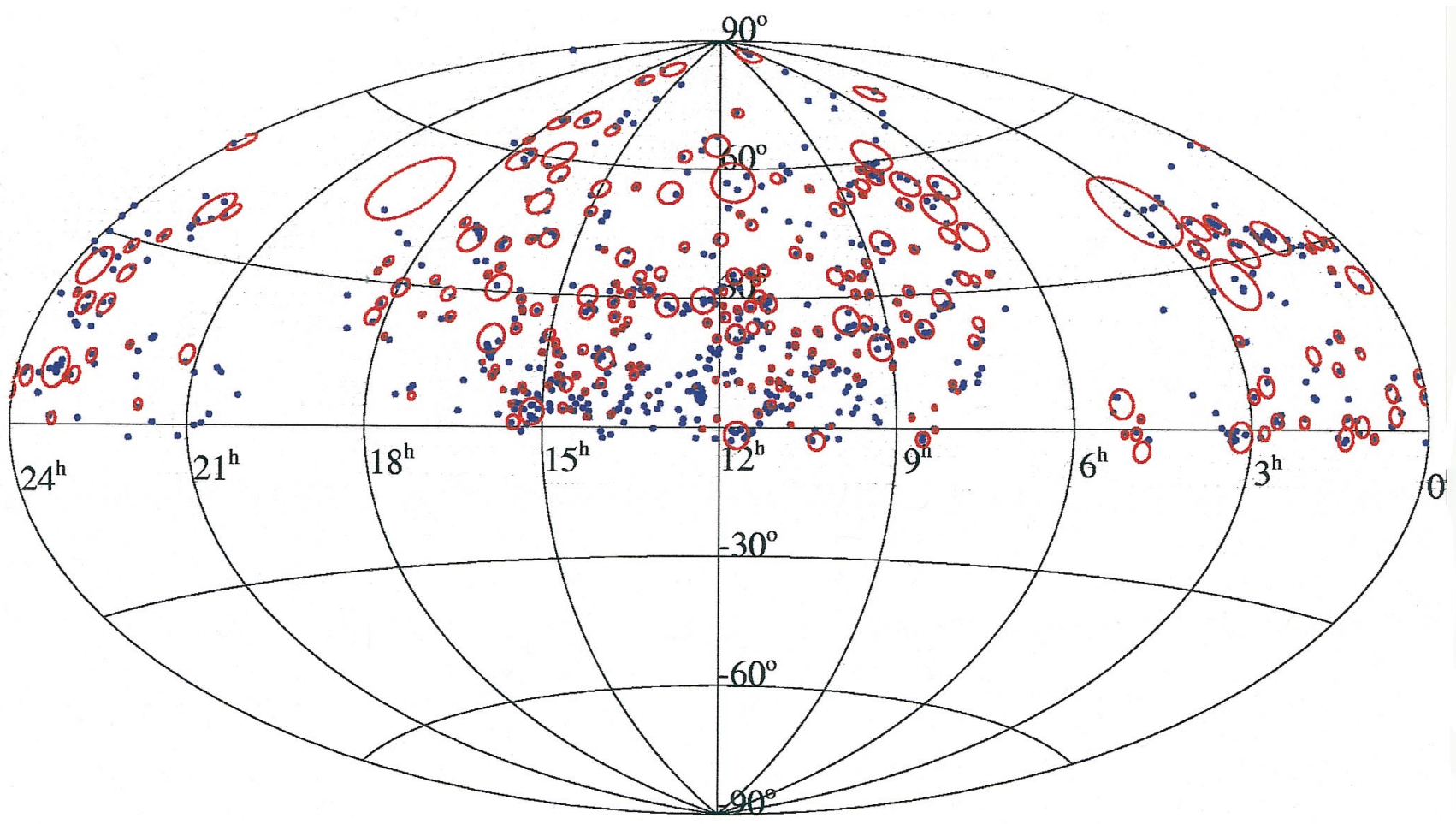

FIG. 1.-Location on the sky of all $732 \mathrm{WBL}$ clusters (blue dots), and all Zwicky clusters (red circles) that contain a poor cluster. The size of the circle corresponds to the Zwicky radius from the CGCG (Table 2, col. [9]). The empty regions at positive declination are due to obscuration by the Galactic plane.

Columns (8)-(10).-Zwicky cluster cross-correlation. Column (8) lists the Zwicky cluster containing the given WBL cluster within its contours. Column $(9)\left(R_{\mathrm{Z}}\right)$ gives the radius of the Zwicky cluster in arcminutes, and column (10) $\left(f_{\mathrm{Z}}\right)$ lists the distance separating the center of the Zwicky and WBL clusters, in units of the Zwicky cluster radius. The radius is that given in the CGCG and is meant to indicate the area on the sky contained within the hand-drawn contours. Zwicky clusters are rarely spherical, so all correlations were verified by eye. Zwicky clusters are also separated into estimated distance classes, with near clusters meant to be in the redshift range $0.0<z \leq 0.05$ and medium-distant clusters in the range $0.05<z \leq 0.1$ (CGCG). We included any near or medium-distant Zwicky cluster that contained a WBL cluster within its contours. Near clusters were chosen over medium-distant clusters in the very few cases where a poor cluster fell within the boundaries of two Zwicky clusters of different distance classes. The letters "MD" attached to a Zwicky cluster name (col. [8]) indicate a medium-distant cluster.

Column (11).- Abell cluster cross-correlation. Any WBL cluster located within one corrected Abell radius (Abell et al. 1989) of a distance class 3 or nearer Abell cluster was considered associated with the Abell cluster. In addition, NED was used to search for WBL cluster members that were also members of Abell clusters.

Column (12).- CfA group cross-correlation. Associated CfA groups were identified as groups whose coordinates were within $30^{\prime}$ of the WBL centroid.

Column (13).--HCG cross-correlation. Associated Hickson compact groups were identified from information on individual galaxies obtained through NED.

Column (14).--Yerkes cluster cross-correlation. The identification of WBL clusters associated with the Yerkes clusters was determined visually as a part of the development of the WBL catalog.

\subsection{Poor-Cluster Galaxies: Table 3}

This table presents basic data on each Zwicky galaxy included as a poor-cluster member. The following information is provided:

Column (1).--Identification. The WBL cluster to which the galaxy belongs.

Columns (2)-(3).-Coordinates. The right ascension and declination of the member galaxy from the CGCG in B1950.0 coordinates.

Column (4).--Magnitude. The apparent photographic magnitude, $m_{\mathrm{pg}}$, of the galaxy from the CGCG.

Columns (5)-(6).- Nearest neighbors. A measure of the number of nearest neighbors to the galaxy at $\sigma_{21}\left(N_{21}\right.$, col. [5]) and $\sigma_{46}\left(N_{46}\right.$, col. [6]). This refers to the number of neighbor galaxies falling within the aperture defining the $\sigma_{21}$ or $\sigma_{46}$ threshold. For a galaxy with no nearest neighbor, the aperture radius is 0.084 at $\sigma_{21}$ and 0.057 at $\sigma_{46}$. The radius scales as $(n+1)^{1 / 2}$, where $n$ is the number of nearest neighbors. For an individual galaxy, the higher the number, the more centrally located it is within the poor cluster. Multiple poor-cluster members with high numbers of neighbors indicate a compact cluster. Galaxies with a nearest neighbor number of zero were merged into a cluster because a nearby galaxy possessed many neighbors. The resulting large aperture $\left[\propto(n+1)^{1 / 2}\right]$ may have overlapped an isolated galaxy in some cases.

Column (7).-Clustering at $\sigma_{46}$. An indication of the fate of each individual galaxy at the higher density enhancement. A blank field indicates that the galaxy became isolated (no neighbors and no overlapping apertures) and 
therefore was not considered a member of a cluster at $\sigma_{46}$. Galaxies with the same letters are part of the same poor cluster at $\sigma_{46}$. WBL designations for these subgroups should include this letter and indicate that it is a $\sigma_{46}$ cluster.

Column (8).-Galaxy redshift. The redshift of the galaxy reported in NED. Several Zwicky galaxies are actually multiple galaxies and therefore have multiple identifications in NED. In these instances, the average of all redshifts available for the Zwicky galaxy is presented.

Column (9).-Galaxy catalog cross-correlations. Cross identifications for the galaxies from the NGC (Dreyer \& Sinnott 1988), UGC (Nilson 1973), and IC (Dreyer \& Sinnott 1988) catalogs obtained from NED. For entries that are actually multiple galaxies, all relevant identifications are presented.

\subsection{Previous Nomenclature: Table 4}

This table cross-references the names of the poor clusters presented here with previous names used in the literature. We also present a direct comparison of the WBL clusters with the Yerkes (AWM, MKW, and WP) poor clusters. We list every Yerkes poor cluster along with any corresponding WBL cluster. If there is no WBL poor cluster associated with a Yerkes cluster, we list a note detailing the reason it was not included (see notes at the end of the table).

\section{DISCUSSION}

Our catalog of poor clusters contains galaxy associations that span several orders of magnitude in the cluster mass function - from very poor systems of only three Zwicky galaxies, up to and including the nearby Abell clusters. Because the number of cluster galaxies listed in Tables 2 and 3 is the number of galaxies brighter than $m_{\mathrm{pg}}=15.7$, it is not necessarily the total membership; fainter galaxies may also be part of these clusters. Further observations are needed to characterize each WBL cluster fully in terms of membership, galaxy morphology, velocity dispersion, etc. From an analysis of a number of the MKW and AWM clusters, Bahcall (1980) showed that these poor clusters are just lower richness extrapolations of Abell clusters in terms of galaxy richness, galaxy density, and spiral fraction (see also Bhavsar 1981). Other subsamples of the WBL catalog indicate similar results in terms of velocity dispersion (Ledlow et al. 1996; Beers et al. 1995), radio source population

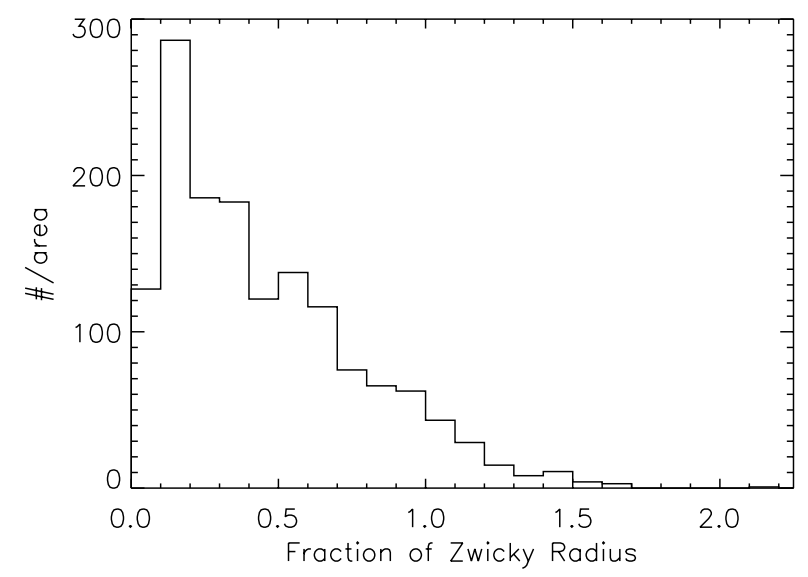

FIG. 2.-Surface number density of all WBL clusters within Zwicky clusters, as a function of the Zwicky radius. WBL poor clusters tend to avoid the centers of Zwicky clusters.
(Burns et al. 1987; Doe et al. 1995), and X-ray properties (Burns et al. 1996; Doe et al. 1995; Price et al. 1991).

The WBL catalog covers the entire sky above $-3^{\circ}$ declination, or approximately $52 \%$ of the sky. The locations of all clusters are shown as dots in Figure 1. Also plotted in Figure 1 are circles representing the Zwicky clusters (near and medium-distant classes) containing one or more WBL clusters. Figure 1 illustrates the interrelatedness of the Zwicky and WBL clusters; most (469 of 732) WBL clusters are high-density galaxy concentrations within the lower density Zwicky cluster contours. Figure 2 shows the locations of WBL poor clusters within the Zwicky clusters. Although the number density of WBL clusters peaks at small radii within the Zwicky contours, most WBL clusters $(\approx 65 \%)$ are located beyond $\frac{1}{2}$ of a Zwicky radius. This indicates subclustering of galaxies within many Zwicky clusters. Subclustering is also displayed in Figure 3, which is a histogram of the number of WBL poor clusters contained within each Zwicky contour. Of the 245 Zwicky clusters that contain WBL poor clusters, $97(\approx 40 \%)$ contain multiple poor clusters.

Some (263) WBL clusters are found outside Zwicky clusters, as more isolated galaxy associations. In fact, there appear to be entire regions of the sky where WBL poor

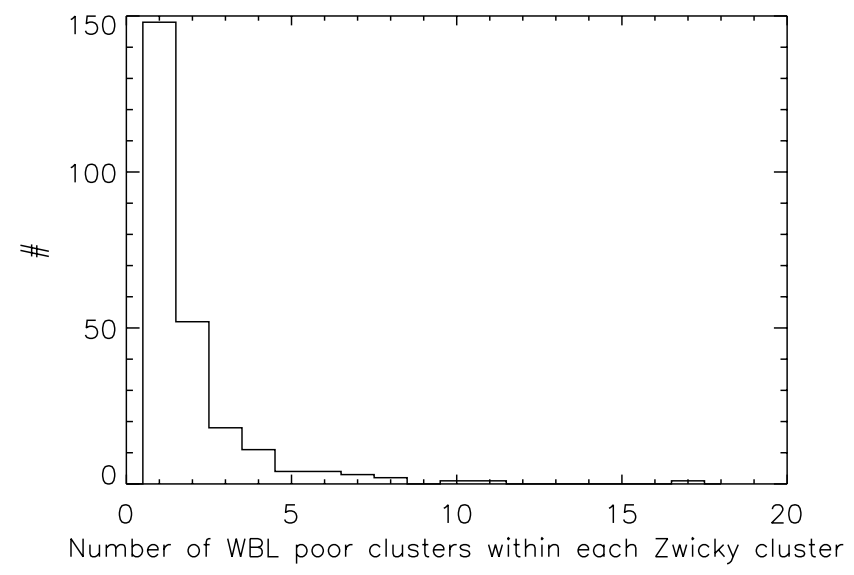

FIG. 3.-Number of WBL clusters within Zwicky clusters. Approximately $40 \%$ of Zwicky clusters that possess WBL clusters contain multiple poor clusters.

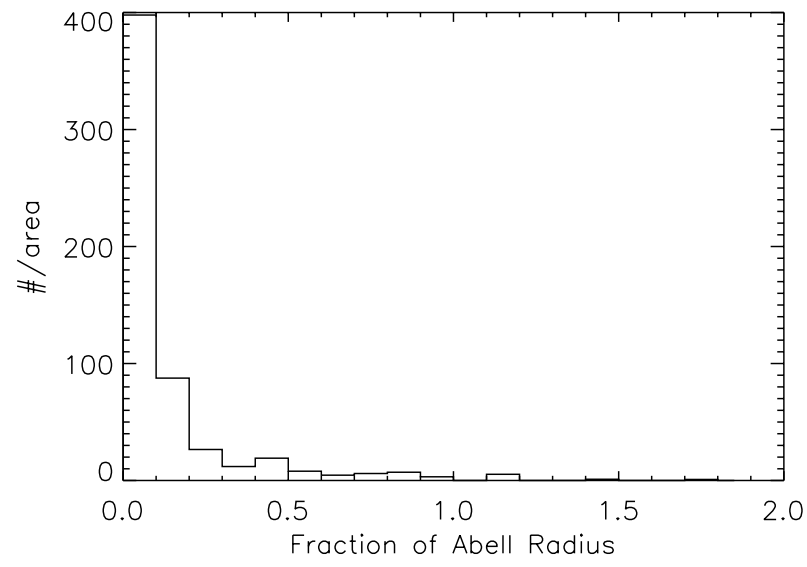

FIG. 4.-Surface number density of all WBL clusters within Abell clusters, as a function of the Abell radius. Most WBL poor clusters are located near the centers of Abell clusters. 
clusters are found but which are devoid of Zwicky clusters (e.g., near $11^{\mathrm{h}}$ R.A., $+10^{\circ}$ decl.; see Fig. 1). In addition, many $(274 / 504 \approx 54 \%)$ of the near Zwicky clusters do not contain WBL clusters. These are generally very loose galaxy associations and are not detected at $\sigma_{21}$. Using a lower $\sigma_{g}$ (such as $10^{1 / 3}$ ), one would detect many more of these Zwicky clusters. Also, the volume limit of the near Zwicky clusters extends beyond the limiting redshift $(z=0.03)$ to which the WBL catalog is complete (see below).

Since the TG algorithm has no upper limit on its richness criterion, the nearby Abell clusters with $\delta>-3^{\circ}$ are also found in our catalog. This was expected and desired, since we wished not only to create a uniform sample of poor clusters but to explore their relation to the rich clusters as well. All distance class (DC) 0 and 1 clusters (a total of 19), and three of the five DC 2 clusters, are detected by the algorithm. The two missing DC 2 Abell clusters have galaxy densities just below the $\sigma_{21}$ detection threshold. We also detect nearly half of the DC 3 Abell clusters with $m_{10}<$ 15.3. Of the 93 Abell clusters with DC $\leq 3$ and $\delta>-3^{\circ}$, $45(\approx 48 \%)$ contain WBL clusters. A distribution of the number density of WBL clusters within Abell clusters is shown in Figure 4, which clearly shows that, unlike the Zwicky clusters, the WBL catalog finds the cores of Abell clusters. This is expected, since Abell clusters are generally more compact than most Zwicky clusters. However, there is still evidence of possible subclustering, as a significant fraction (35\%) of the Abell clusters coincident with our catalog contain multiple WBL clusters (Fig. 5).

As expected, the WBL catalog also has significant overlap with other group catalogs, such as the HCG and CfA groups, but because of the differing selection algorithms there is no direct correlation. Although the cross identifications are not listed in Table 2, the WBL catalog also detects a number of TG groups, since the same algorithm was used to find both sets of poor clusters. TG searched to much lower galaxy densities $\left(10^{2 / 3}\right)$, and used a brighter magnitude cutoff $\left(m_{\mathrm{pg}}<14\right)$, therefore focusing on very nearby, looser galaxy associations in the north Galactic cap $\left(b>40^{\circ}, \delta>0^{\circ}\right)$.

The completeness of the entire WBL catalog is difficult to determine. Certainly, the portions of the catalog at very low Galactic latitudes $\left(|b|<30^{\circ}\right)$ are incomplete as a consequence of obscuration by the Galactic plane. Also, since

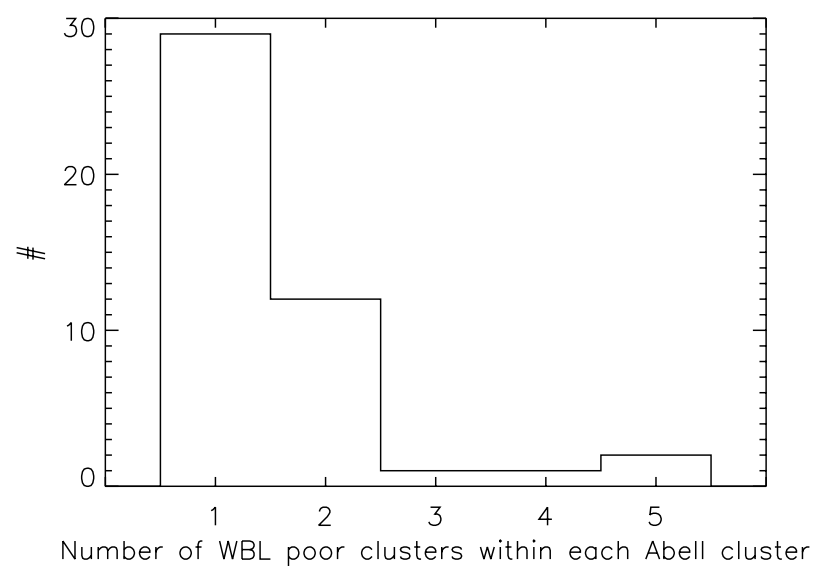

FIG. 5.- Number of WBL clusters within Abell clusters. Approximately $35 \%$ of Abell clusters that possess WBL clusters contain multiple poor clusters.

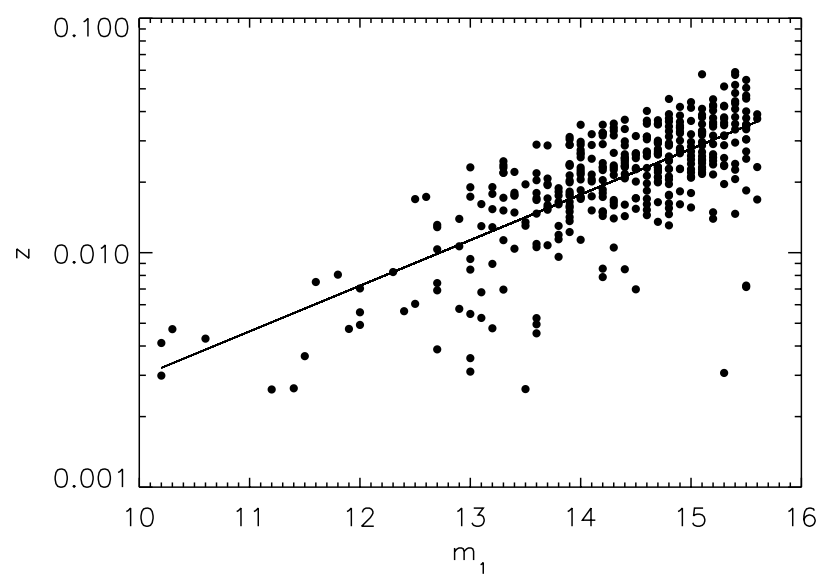

FIG. 6.-Relation used to estimate redshifts, $z$, from the apparent magnitude of the brightest cluster member, $m_{1}$.

there is no redshift information for a large number of the galaxies in our catalog, a discussion of completeness, and the frequency of projection effects, can only be approximate (see Burns et al. 1996 for a discussion of projection effects in a subsample of the catalog). For poor clusters without welldetermined mean redshifts, we estimated redshifts based on the magnitude of the brightest cluster member $\left(m_{1}\right)$. We have used poor clusters from Table 2 , with no redshift notes (col. [8]), and thus reasonably well determined redshifts, to calibrate the $m_{1}-z$ relation for the remaining poor clusters. Included in this calibration are poor clusters with greater than seven reported redshifts, containing a (iii) in column (8), which are certainly physical systems along with a few outlying galaxies. This relation, using 394 poor clusters, is shown in Figure 6. From the redshifts in Table 2 combined with the estimated redshifts for all other clusters, we have determined the volume density of the WBL catalog for $|b|>30^{\circ}$. In Figure 7 we show the volume density in redshift bins of $\Delta z=0.005$. Based on this analysis, this sample is nearly volume limited over the redshift range

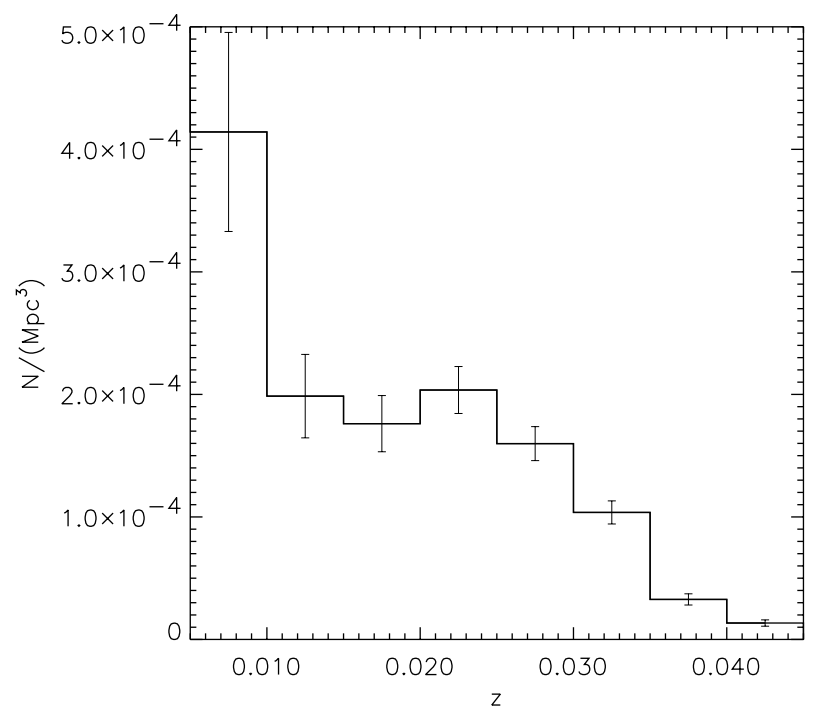

FIG. 7.- Volume density of WBL poor clusters in the Galactic latitude range $|b|>30^{\circ}$. We judge this poor-cluster sample to be volume limited in the redshift range $0.01<z<0.03$. Error bars represent Poisson errors in each bin. 
$0.01<z<0.03$. A similar result was found for a smaller sample of WBL clusters in Ledlow et al. (1996). We are employing the volume-limited portion of the WBL catalog $\left(|b|>30^{\circ}, 0.01<z<0.03\right)$ for an extensive X-ray, optical, and radio study of poor clusters.

\section{CONCLUSIONS}

We present a catalog of 732 optically selected poor clusters of galaxies. These WBL poor clusters were identified as galaxy surface density enhancements in the CGCG, which cataloged galaxies brighter than photographic magnitude 15.7, and declination greater than $-3^{\circ}$. The WBL catalog covers a wide range of cluster richness, from very poor systems containing only a few galaxies, through the Yerkes poor clusters, and including many nearby Zwicky and Abell clusters. Previous analyses of the WBL clusters show a number of them contain an X-ray-bright intracluster medium (Burns et al. 1996; Price et al. 1991) and interesting extragalactic radio morphologies (Burns et al. 1987; Doe et al. 1995) similar to rich clusters. WBL clusters with $|b|>30^{\circ}$ are nearly volume limited in the redshift range $0.01<z<0.03$ (Fig. 7), producing a subsample of $\approx 300$ systems that is ideal for studying the properties of poor clusters. Taken in its entirety, the WBL catalog covers over 3 orders of magnitude in the cluster mass function (Burns et al. 1996) and provides an excellent sample with which to study the formation and evolution of a wide range of galaxy associations.

This work was completed with the assistance of NSF grant AST 98-6039 and NASA grant NAG 5-6772 to J. O. B. and M. B.; S. P. B. wishes to acknowledge the Kentucky Space Grant Consortium for its support, including subgrant WKU 521781-97-02. P. L. B.'s contribution was supported by the NRAO Summer Student Program. Shortly before this paper was submitted, the lead author, Richard A. White, passed away following a long and valiant struggle with leukemia. We, his coauthors, friends, and colleagues, wish to dedicate this paper to Richard. His love for and devotion to astronomy continue to inspire us all.
Abell, G. O. 1958, ApJS, 3, 211

Abell, G. O., Corwin, H. G., Jr., \& Olowin, R. P. 1989, ApJS, 70, 1

Albert, C. E., White, R. A., \& Morgan, W. W. 1977, ApJ, 211, 309

Bahcall, J. N. 1980, ApJ, 240, 377

Beers, T. C., Kriessler, J. R., Bird, C. M., \& Huchra, J. P. 1995, AJ, 109, 874

Bhavsar, S. P. 1980, ApJ, 237, 671

-1981, ApJ, 246, L5

Bhavsar, S. P., \& Piggott, R. G., Jr. 1983, ApJS, 52, 23

Burns, J. O., Hanisch, R. J., White, R. A., Nelson, E. R., Morrisette, K. A., \& Moody, J. W. 1987, AJ, 94, 587

Burns, J. O., Ledlow, M. J., Loken, C., Klypin, A., Voges, W., Bryan, G. L., Norman, M. L., \& White, R. A. 1996, ApJ, 467, L49

Doe, S. M., Ledlow, M. J., Burns, J. O., \& White, R. A. 1995, AJ, 110, 46

Dreyer, J. L. E., \& Sinnott, R. W. 1988, NGC 2000.0 (Cambridge: Sky

Publishing)

\section{REFERENCES}

Geller, M. J., \& Huchra, J. P. 1983, ApJS, 52, 61

Hickson, P. 1982, ApJ, 255, 382

Ledlow, M. J., Loken, C., Burns, J. O., Hill, J. M., \& White, R. A. 1996, AJ, 112,388

Morgan, W. W., Kayser, S., \& White, R. A. 1975, ApJ, 199, 545

Nilson, P. 1973, Uppsala General Catalogue of Galaxies (Uppsala: Uppsala Astron. Obs.)

Price, R., Duric, N., Burns, J. O., \& Newberry, M. V. 1991, AJ, 102, 14

Turner, E. L., \& Gott, J. R., III. 1976, ApJS, 32, 409 (TG)

White, R. A. 1978, ApJ, 226, 591

Zwicky, F., Herzog, E., Karpowicz, M., Kowal, C. T., \& Wild, P. 19611968, Catalogue of Galaxies and of Clusters of Galaxies (6 vols.; Pasadena: Caltech) 